ARTICLE

https://doi.org/10.1038/s41467-020-19884-6 OPEN

\title{
A nanobody suite for yeast scaffold nucleoporins provides details of the nuclear pore complex structure
}

Sarah A. Nordeen (1D ${ }^{1}$, Kasper R. Andersen (10 ', Kevin E. Knockenhauer ${ }^{1}$, Jessica R. Ingram², Hidde L. Ploegh (iD ${ }^{2}$ \& Thomas U. Schwartz (i) ${ }^{1 凶}$

Nuclear pore complexes (NPCs) are the main conduits for molecular exchange across the nuclear envelope. The NPC is a modular assembly of $\sim 500$ individual proteins, called nucleoporins or nups. Most scaffolding nups are organized in two multimeric subcomplexes, the Nup84 or Y complex and the Nic96 or inner ring complex. Working in S. cerevisiae, and to study the assembly of these two essential subcomplexes, we here develop a set of twelve nanobodies that recognize seven constituent nucleoporins of the $Y$ and Nic96 complexes. These nanobodies all bind specifically and with high affinity. We present structures of several nup-nanobody complexes, revealing their binding sites. Additionally, constitutive expression of the nanobody suite in S. cerevisiae detect accessible and obstructed surfaces of the $Y$ complex and Nic96 within the NPC. Overall, this suite of nanobodies provides a unique and versatile toolkit for the study of the NPC.

\footnotetext{
${ }^{1}$ Department of Biology, Massachusetts Institute of Technology, Cambridge, MA, USA. ${ }^{2}$ Boston Children's Hospital and Harvard Medical School, Boston, MA, USA. ${ }^{\bowtie}$ email: tus@mit.edu
} 
T he hallmark of the eukaryotic cell is a complex endomembrane system of organelles that compartmentalize specific functions within the cell. One of the largest of these organelles is the nucleus, which stores the genetic material and is the site of replication, transcription, and ribosome synthesis. Soluble transport of molecules into and out of the nucleus occurs solely through nuclear pore complexes (NPCs), 40-120 MDa ring-like channels that perforate the inner and outer membranes of the nuclear envelope (NE) (Fig. 1a). Roughly 30 nucleoporins (nups) contribute to the modular, eightfold symmetric assembly of subcomplexes that comprise the $\mathrm{NPC}^{1-4}$. One of the main structural subcomplexes is the Nup84 or Y complex. In S. cerevisiae, the $575 \mathrm{kDa} \mathrm{Y}$ complex has seven components, many of which are essential or produce severe phenotypes when deleted $^{5-7}$. The Y complex structure consists of two short arms that meet a long stalk at a central triskelion-like hub ${ }^{8-10}$ (Fig. 1b). The other main structural component of the NPC is the heteromeric Nic96 or inner ring complex ${ }^{11}$. This $\sim 0.5 \mathrm{MDa}$ complex occupies the inner ring of the NPC and anchors the trimeric Nsp1 complex, which contains three phenylalanine-glycine (FG)-nups that are essential for maintaining the permeability barrier ${ }^{11-16}$. Both Y complex nups (Nup84, Nup85, and Nup145C) and Nic96 have an ancestral coatomer element 1 (ACE1) fold, conserved across the NPC and COPII vesicle coats ${ }^{17,18}$. The tripartite fold consists of crown, trunk, and tail modules and the interfaces between each module act as somewhat flexible joints. How this flexibility affects the NPC assembly is still unclear.

Its size, flexibility, and membrane interactions pose challenges for the elucidation of the structure of the NPC. But only with detailed structural information will we obtain mechanistic insight into the many functions of NPCs. To arrive at a structure, different labs approach the problem by either a bottom-up or topdown approach. For the bottom-up approach, the many modular structural assemblies that make up the NPC have been broken down into further sub-assemblies which are then studied primarily by X-ray crystallography. Over the past decade, many of the structural elements have been characterized (reviewed in refs. ${ }^{2,19}$ ). We now have a complete composite model for the Y complex from S. cerevisiae ${ }^{20}$. For the top-down approach, cryoelectron tomography (cryo-ET) has been used to visualize whole NPCs while still embedded in the nuclear membrane or after detergent extraction. Recent studies have yielded maps of the entire NPC at 2-5 nm resolution for human, Xenopus laevis, and S. cerevisiae ${ }^{21-24}$. The resolution gap between the top-down and bottom-up approaches has narrowed. Along with multiple stoichiometry studies, docking of the many crystal structures of nups into the cryo-ET maps has been attempted ${ }^{22,23,25-28}$. For S. cerevisiae, the cryo-ET map allows placement of $\mathrm{Y}$ complexes into the density, resulting in a model that contains a total of 16 copies per $\mathrm{NPC}^{24}$. This model consists of two eight-membered rings, one each on both the cytoplasmic and nucleoplasmic faces of the $\mathrm{NPC}^{23,24}$. The $\mathrm{Y}$ complexes are arranged in a head-to-tail manner, with the main interface mediated by Nup120 and Nup $133^{29}$. On the cytoplasmic face, the Nup82 complex anchors to the Y complex via Nup8530. Thirty-two copies of Nic96 are nestled tightly into the inner ring complex along with Nup192, another scaffolding nup ${ }^{23}$. While these studies improved our understanding of the overall architecture of the NPC, the resolution of these cryo-ET maps does not reveal secondary structure. This leaves room for further interrogation and improvement. We aimed to create a set of tools that will aid in studying the complicated NPC assembly in more detail.

Here we describe a nanobody library comprising 12 unique nanobodies to the $\mathrm{Y}$ complex and Nic96 from S. cerevisiae. Nanobodies are single-domain (VHH) antibody fragments derived from camelid heavy-chain only antibodies. Nanobodies are excellent tools both in vitro and in vivo, as they are small $(\sim 14 \mathrm{kDa})$, easily purified from E. coli, easily modified with fluorophores, and typically have nanomolar binding affinities ${ }^{31,32}$. The library consists of nanobodies that bind to each of the 6 conserved nups in the Y complex ${ }^{8}$. We describe their in vitro binding characteristics using bio-layer interferometry, size-exclusion chromatography (SEC), and, in several cases, their nup-bound structures by X-ray crystallography. We show the effects of nanobody expression in vivo and how these results suggest accessible and inaccessible surfaces within the assembled NPC. Together, this work provides a toolkit for studying the scaffold of the NPC and uncovers details of the NPC structure in vivo.

\section{Results}

A nanobody library to the $\mathrm{Y}$ complex and Nic96. In order to generate nanobodies specific to NPC scaffold nups, we separately immunized alpacas with recombinantly purified full-length Y complex and Nic96. We then selected nanobodies by phage display using single nups or subassemblies of the $\mathrm{Y}$ complex as targets (Fig. 1b). This allowed us to obtain nanobodies that cover both short arms, the hub, and the long stalk of the Y complex. We thus compiled a set of twelve nanobodies that bind Y complex nups and Nic96 (Fig. 1b, c). The library covers a wide range of sequence space (Fig. 1c). Both, the sequences and lengths of complementarity determining regions (CDRs) 1 and 2, are more similar than CDR3 across the library. However, there is no common CDR across the set. The greatest differences arise in CDR3, which varies from 9 to 24 residues. This wide deviation in length is attributed to the vast genetic diversity contributed by the immunized alpaca, rather than the typically shorter and invariant lengths used in in vitro selection methods ${ }^{33,34}$.

After selection, we obtained two nanobodies (VHH-SAN6 and 7) that recognize a truncated Y complex hub construct (Nup120 and Nup85 C-terminal domains, full-length Nup145C-Sec13) (Fig. 1b). In order to identify the nups these nanobodies target, we first tested binding by SEC with full-length nups (Nup120, Nup85, and a fusion construct of Nup145C-Sec13 ${ }^{35}$ ). We found that both bound Nup145C-Sec13. To further narrow down the binding site, we conducted a second SEC experiment using only Sec13 fused to the Nup145C insertion blade ${ }^{35}$ and found that VHH-SAN7 recognizes Sec13 rather than Nup145C (Supplementary Fig. 1). VHH-SAN6 did not bind Sec13 alone, meaning that it recognizes Nup145C. Sec13 is tightly packed within the hub of the Y complex, so we tested the ability of VHH-SAN7 to bind the assembled Y complex hub. When pre-incubated with Nup120-Nup85-Seh1-Nup145C-Sec13, VHH-SAN7 co-elutes as a heterohexameric complex (Supplementary Fig. 1). This experiment suggests that VHH-SAN7 binds Sec13 in the context of the assembled $\mathrm{Y}$ complex.

Three nanobodies (VHH-SAN1/2/3) in the library recognize Nup85. As an ACE1 nup, we examined whether these nanobodies bind to one of three modules (crown, trunk, tail) within the domain. By SEC analysis we found that all three nanobodies bound Nup85 trunk-crown -Seh1 (Supplementary Figs. 2, 3). We then tested the binding of each nanobody to Nup85 crown, which formed a stable complex with VHH-SAN2 and 3, but not VHHSAN1 (Supplementary Fig. 2). Binding data suggest that VHHSAN2 and 3 recognize distinct, non-overlapping epitopes, as we observe a heptameric complex of Nup85 trunk-crown-Seh1-VHHSAN2/3 by SEC (Supplementary Fig. 2). We verified that VHHSAN1 bound Nup85 by observing no complex formation with Seh1 alone (Supplementary Fig. 3).

Nanobodies bind with varying kinetics, but strong affinities. In order to characterize the binding kinetics of our nanobody 
a

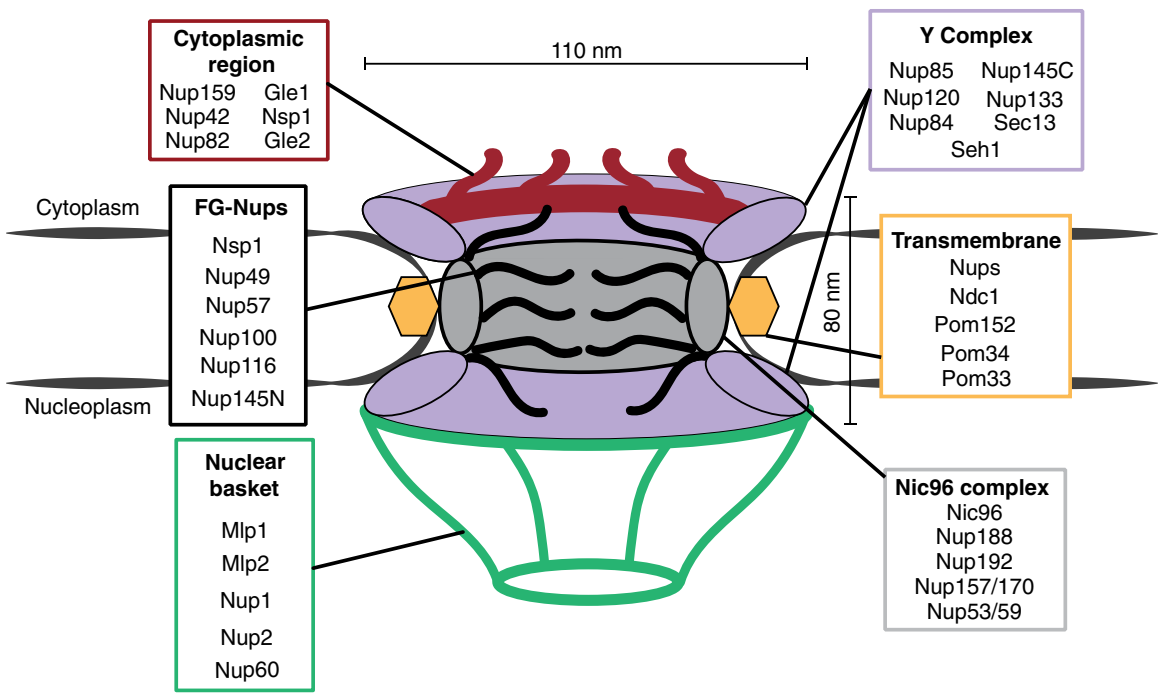

b Injected antigen

Selection fragments

Nanobody library
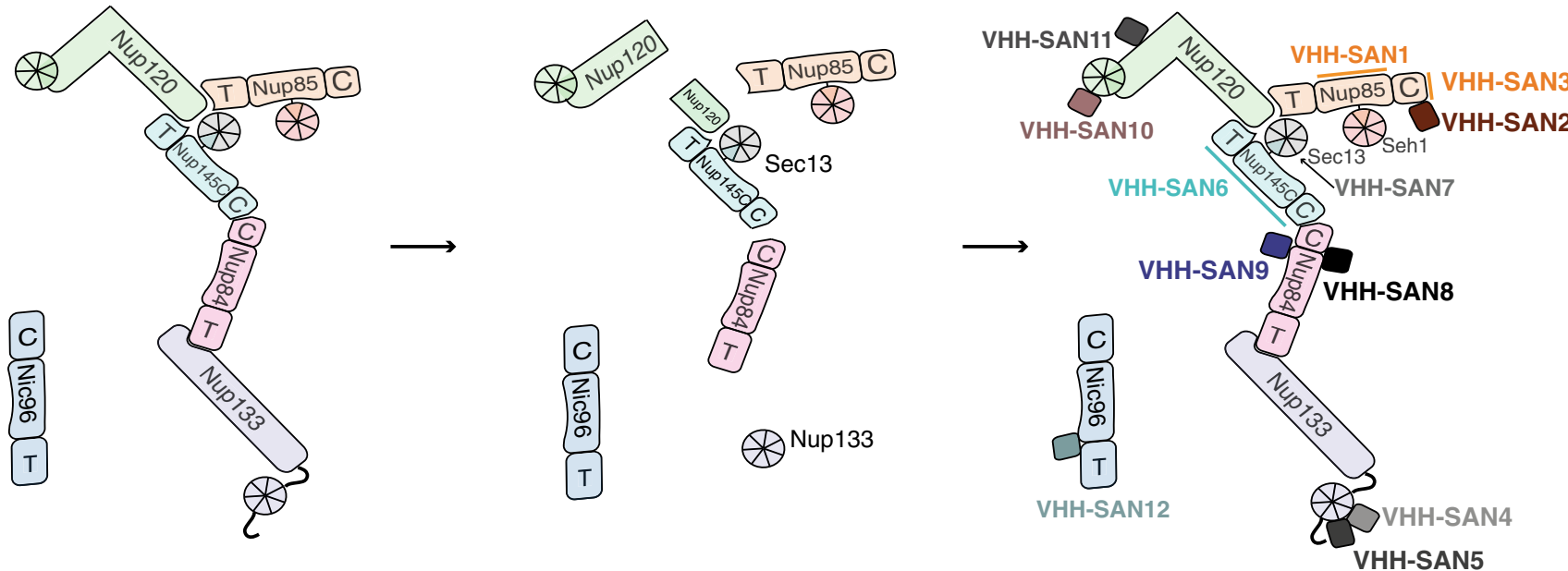

C

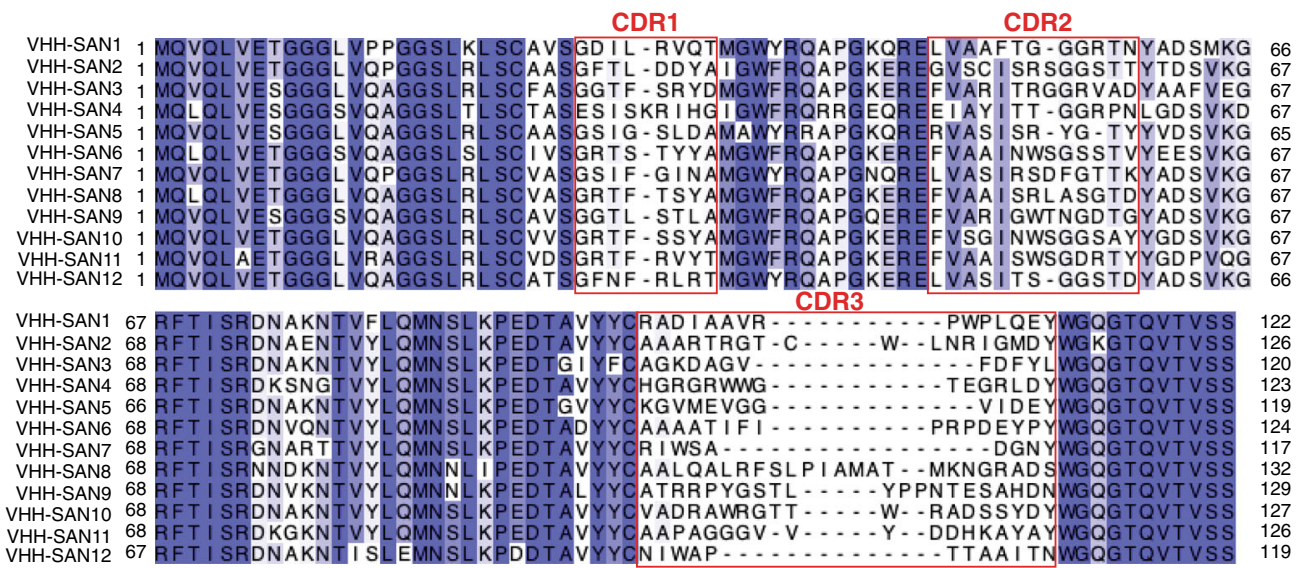

Fig. 1 A nanobody library to scaffold nucleoporins. a Schematic of the NPC assembly and classification of yeast nups. Each subcomplex or type of nup is listed together in a box and the relative position within the NPC assembly is indicated. $\mathbf{b}$ Schematic of the Y complex and Nic96 used for alpaca immunization, selection by phage display, and resulting nanobody library. Elements of ACE1 fold proteins are indicated: T-tail and C-crown adjacent to the central trunk element. c Sequence alignment of the nanobody library, colored by percentage identity. Variable complementarity determining regions (CDRs) are indicated.

library, we employed bio-layer interferometry (BLI). We affixed each nanobody with a C-terminal biotinylated Avi-tag ${ }^{36}$ to streptavidin-coated biosensor tips. We assayed binding to each nanobody's respective nup target and observed a variety of binding kinetics across the nanobody library (Table 1, Fig. 2). The tightest binder, VHH-SAN3, dissociates very slowly resulting in a binding constant of $\sim 14$ pM. In fact, a slow dissociation rate applies to the majority of the library. A few nanobodies, 
VHH-SAN2, 3, 8, and 9, have off rates less than $\sim 1 \times 10^{-4} \mathrm{~s}^{-1}$ resulting in very tight equilibrium binding constants (14-170 pM). Of the set, VHH-SAN4 has the comparatively weakest affinity of $\sim 230 \mathrm{nM}$, due to a much faster off rate than the other members the library and is the only nanobody that has an equilibrium binding constant of less than $\sim 10 \mathrm{nM}$. Overall, the nanobodies bind very stably to their antigens.

Mapping of nanobody epitopes by X-ray crystallography. We mapped the epitopes of eight of the twelve nanobodies by solving the crystal structures of multiple nup-nanobody complexes. This enabled us to visualize the binding epitopes in greater detail. We solved the structures of nups that cover both short arms and the stalk of the Y complex: Nup85-Seh1-VHH-SAN2, Nup120 ${ }_{1-757^{-}}$ VHH-SAN10/11, and Nic96 ${ }_{186-839}-\mathrm{VHH}-\mathrm{SAN} 12$. We used

Table 1 Nanobody-binding affinities.

\begin{tabular}{|lllll} 
Nanobody & Nucleoporin & $\boldsymbol{K}_{\mathbf{D}}(\mathbf{M})$ & $\boldsymbol{k}_{\text {on }}\left(\mathbf{M}^{-1} \mathbf{s}^{-\mathbf{1}}\right)$ & $\boldsymbol{k}_{\text {off }}\left(\mathbf{s}^{-\mathbf{1}}\right)$ \\
\hline VHH-SAN1 & Nup85 & $5.8 \times 10^{-10}$ & $2.3 \times 10^{6}$ & $1.4 \times 10^{-3}$ \\
VHH-SAN2 & & $8.0 \times 10^{-11}$ & $1.0 \times 10^{6}$ & $8.1 \times 10^{-5}$ \\
VHH-SAN3 & & $1.4 \times 10^{-11}$ & $5.8 \times 10^{5}$ & $8.1 \times 10^{-6}$ \\
VHH-SAN4 & Nup133 & $2.3 \times 10^{-7}$ & $9.5 \times 10^{5}$ & $2.2 \times 10^{-1}$ \\
VHH-SAN5 & & $1.0 \times 10^{-8}$ & $2.8 \times 10^{5}$ & $2.8 \times 10^{-3}$ \\
VHH-SAN6 & Nup145C & $2.4 \times 10^{-10}$ & $5.3 \times 10^{5}$ & $1.3 \times 10^{-4}$ \\
VHH-SAN7 & Sec13 & $5.1 \times 10^{-10}$ & $1.3 \times 10^{6}$ & $6.5 \times 10^{-4}$ \\
VHH-SAN8 & Nup84 & $6.3 \times 10^{-11}$ & $3.5 \times 10^{5}$ & $2.2 \times 10^{-5}$ \\
VHH-SAN9 & & $1.7 \times 10^{-10}$ & $4.4 \times 10^{5}$ & $7.7 \times 10^{-5}$ \\
VHH-SAN10 & Nup120 & $3.8 \times 10^{-10}$ & $4.5 \times 10^{5}$ & $1.7 \times 10^{-4}$ \\
VHH-SAN11 & \multirow{2}{*}{ VHH } & $7.9 \times 10^{-10}$ & $9.8 \times 10^{5}$ & $7.8 \times 10^{-4}$ \\
VHH-SAN12 & Nic96 & $1.3 \times 10^{-9}$ & $1.8 \times 10^{6}$ & $2.4 \times 10^{-3}$ \\
\hline
\end{tabular}

VHH-SAN4/5 and VHH-SAN8/9 as crystallization chaperones for the previously uncharacterized Nup133 $3_{\text {NTD }}$ and Nup84Nup133 CTD complex structures, respectively (see the accompanying paper, https://doi.org/10.1101/2020.06.19.161133). VHH-SAN4 and five bind adjacent epitopes on the same face of the Nup133 ${ }_{\text {NTD }} \beta$-propeller. Both, VHH-SAN4 and VHH-SAN5, contributed significant packing interfaces in the crystal lattice, facilitating high-resolution diffraction of a previously elusive target. In addition, both nanobodies rely primarily on CDR3 to recognize Nup133, with VHH-SAN4 making no contacts with either its CDR1 or 2. This is perhaps why VHH-SAN4 has the lowest binding affinity of the library, as CDRs 1 and 2 typically enhance binding strength ${ }^{37}$. Like Nup85, Nup145C, and Nic96, Nup84 has an ACE1 domain fold. Interestingly, both Nup84specific nanobodies (VHH-SAN8 and 9) bind at the crown-trunk module interface, recognizing opposite faces of Nup84. It is not unusual for nanobodies to bind to a domain-domain interface ${ }^{38}$. While our attempts at co-crystallization of VHH-SAN1, 3, 6, and 7 with their nup targets failed, we were able to narrowly define their binding sites using SEC with smaller nup constructs.

We solved Nup85 trunk-crown-Seh1 in complex with VHH-SAN2 by molecular replacement (MR) using the structure of scNup85 $564^{-S e h} 1$ as a search model ${ }^{18}$ (Fig. 3). From previous biochemical experiments, we expected that VHH-SAN2 binds the Nup85 crown module (Supplementary Fig. 2). After examining the Nup85 crown in the electron density map, we observed additional difference density, but not sufficient to encompass an entire nanobody (Supplementary Fig. 4). This made it difficult to place the nanobody by MR. We generated a VHH-SAN2 model using SWISS-MODEL ${ }^{39}$ and manually placed it into the difference density map (Supplementary Fig. 4). We used a nanobody model that included the CDR loops, as these residues are most likely what make up the density closest to Nup85. After several rounds

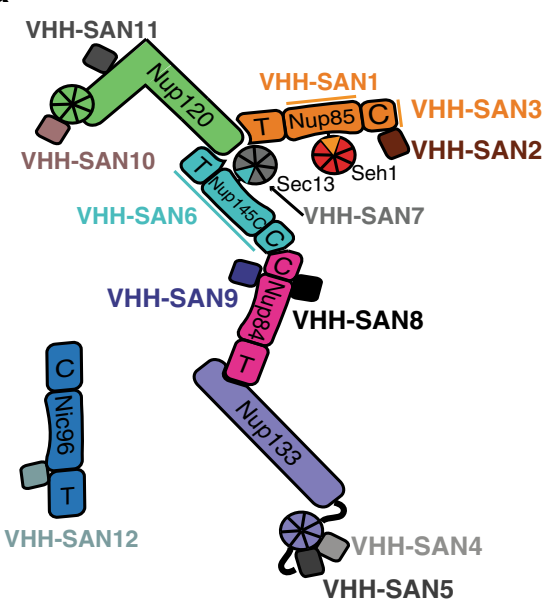

b Nup85

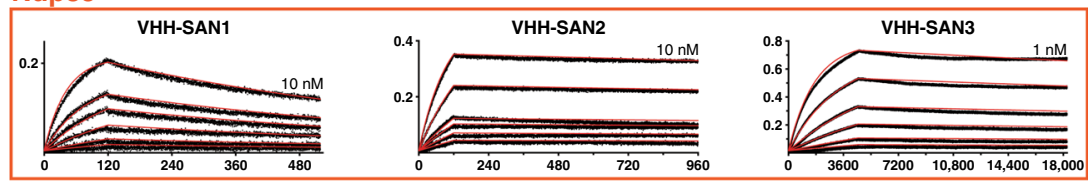

Nup133

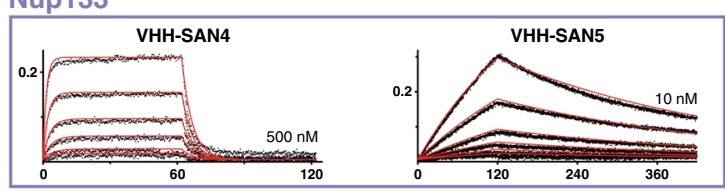

Nup145C

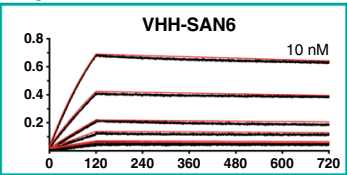

Sec13

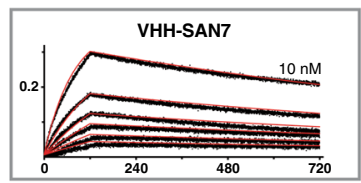

Nup84
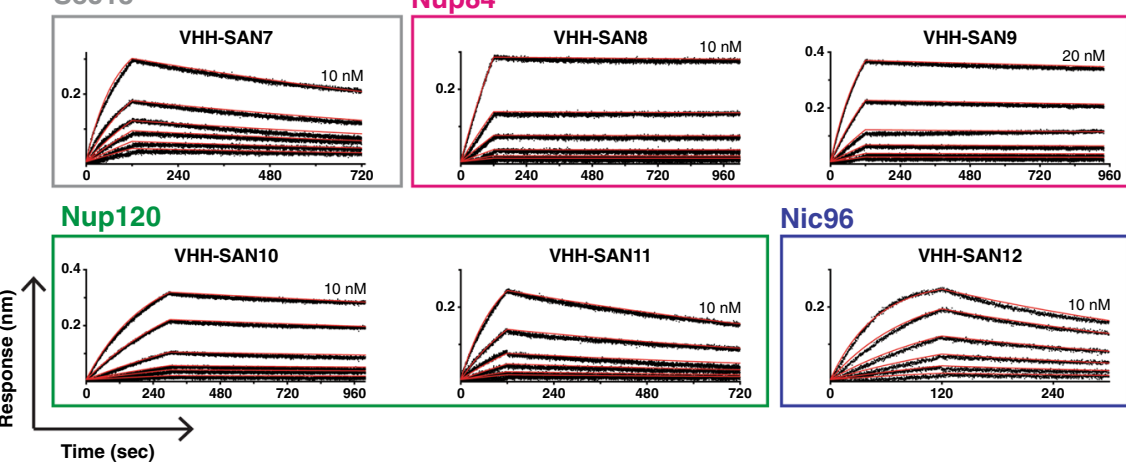

Nic96

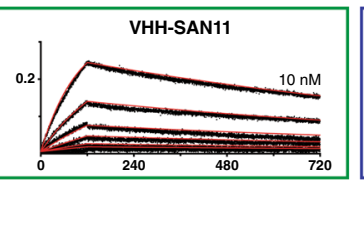

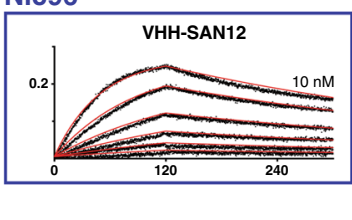

Fig. 2 Bio-layer interferometry of nanobody-nup binding. a Schematic of the $Y$ complex and Nic96 with the nanobody library. b Binding curves showing association and dissociation kinetics for each nanobody-nup pair. Nanobodies with a biotinylated C-terminal Avi-tag were fixed (ligand) and nups were used as analytes. Curves were corrected for buffer background. Each set of curves is a twofold dilution series from the highest concentration listed on each plot. Experimental data are shown as black dotted lines with red lines indicating globally fitted curves. Axis labels for all plots are indicated in the bottom left. 


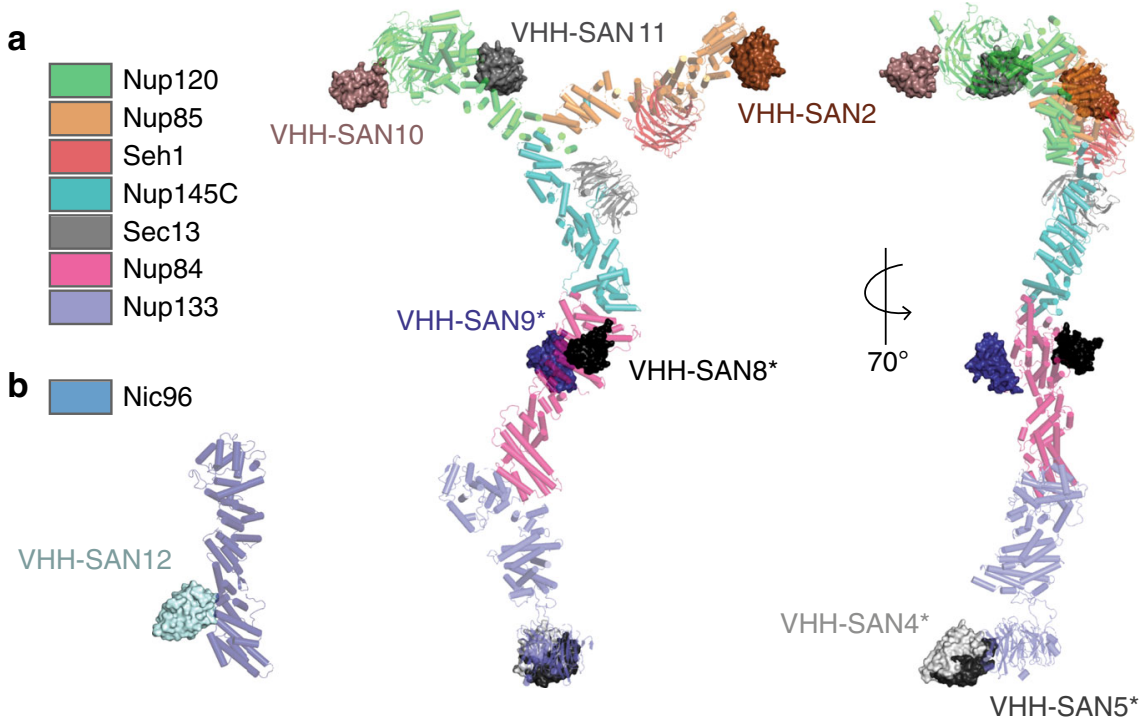

Fig. 3 Structures of nanobodies complexed to the $\mathbf{Y}$ complex and Nic96. a Structures of nanobody-nup complexes docked onto the composite structure of the $\mathrm{Y}$ complex. Nanobodies are shown as surfaces, the $\mathrm{Y}$ complex is shown as cartoon. VHH-SAN10/11 was solved in complex with $\mathrm{Nup}^{2} \mathrm{O}_{1-757}$. VHHSAN2 was solved in complex with Nup85-Seh1 ${ }_{1-564}{ }^{*}$ Indicates structures described in ref. ${ }^{20}$. b Structure of Nic96 in complex with VHH-SAN12.

of positional refinement, the nanobody fit reasonably well into the density (Supplementary Fig. 4).

VHH-SAN10 recognizes a flexible loop in Nup120. We also solved the complex of Nup120 $1-757^{-V H H}-\mathrm{SAN} 10 / 11$ by MR using the published scNup120 $1-757$ structure as a model ${ }^{40}$. After initial refinement, we observed additional density near residues 431-439 of Nup120, which we could attribute to a nanobody using MR. However, there was no obvious additional density for a second nanobody. Analysis of the crystals by SDS-PAGE showed that both nanobodies were present in the crystal (Supplementary Fig. 5). We then examined the crystal packing to see if there was space to accommodate the missing nanobody adjacent to unstructured loops. We hypothesized that if the nanobody was completely missing in the density, the epitope was also disordered in the crystal and therefore missing in the density as well. There are five unstructured loops in the $\beta$-propeller domain of Nup120 that neighbor solvent channels (Supplementary Fig. 5). A nanobody's dimension are roughly $35 \AA \times 15 \AA \times 15 \AA$. Only one large loop, residues 187-203, faces a solvent channel in the crystal that appeared sufficiently large to accommodate a nanobody. We hypothesized that the missing nanobody bound this loop and tested whether replacement of residues 187-203 with a flexible linker (GGSx5) would ablate binding by BLI. Indeed, we found that VHH-SAN10 no longer bound this Nup120 mutant, but as expected, VHH-SAN11 still recognized Nup120 (Fig. 4c). In a reciprocal experiment, we replaced residues 431-439 with a flexible linker (GSSx3) and tested VHH-SAN10 versus VHHSAN11 binding. This experiment confirmed that VHH-SAN11, and not VHH-SAN10, binds to the 431-439 region (Fig. 4b).

Both VHH-SAN10 and 11 are interesting nanobodies, as they recognize relatively unstructured regions of Nup120. Typically, nanobodies recognize structured regions or clefts in their target, owing to their long CDR loops that prefer to insert into and along concave surfaces $^{38}$. Regardless of this difference, both have very high binding affinities (Table 1). VHH-SAN10 and 11 add to a growing list of nanobodies that bind to short epitopes that function outside of a folded domain ${ }^{41-44}$.

VHH-SAN12 binds Nic96 between its trunk and tail modules. In addition to the $\mathrm{Y}$ complex nanobodies, we identified a nanobody, VHH-SAN12, that binds Nic96 of the inner ring complex. We solved the complex of Nic96 ${ }_{186-839}$ with VHHSAN12 by MR with the published scNic $96_{186-839}$ structure as a template (Fig. 5a) ${ }^{45}$. Nic96 $186-839$ forms an elongated structure of 30 a-helices. Overall, the dimensions, shape, and ACE1 fold of Nic96 are identical to the previously described structures ${ }^{45,46}$. The $\mathrm{N}$ terminus is in the center of the protein that then zig-zags towards one end of the molecule. Helices a4-12 fold over themselves, forming the crown of the ACE1 domain, with a6-9 running perpendicular to the trunk helices of a13-21. The Cterminal helices $a 22-30$ form the tail and zig-zag away from the trunk at an angle. VHH-SAN12 inserts its CDR loops 1 and 2 into the space between helices $\alpha 20-21$ and $\alpha 22-25$. Interestingly, VHH-SAN12 has one the shortest CDR3 of the library and CDR3 contributes little to the binding interface, which is unusual for a nanobody ${ }^{37}$. Even without this contribution, VHH-SAN12 has a high affinity for Nic96 (Fig. 2, Table 1).

Helices a 20-25 delineate the trunk and tail interface of the ACE1 fold. In comparison to the previously solved structures, we observe a change in conformation. Aligning the tail domains of this structure and the structure from Jeudy et al. ${ }^{45}$, we observe a kink that translates through the remainder of the molecule (Fig. 5b). VHH-SAN12 brings helices $\alpha 20-21$ and $\alpha 22-25$ slightly closer to each other, resulting in a $\sim 19 \AA$ shift between the two crown domains. This shift is slightly smaller $(\sim 10 \AA)$ when comparing our structure to the one described in Schrader et al. ${ }^{46}$. While the overall conformation changes, the three modules of the ACE1 fold individually superpose very well. In comparing the crown domains, our new structure is much better defined (Fig. 5c). At the increased resolution (from 2.5 to $2.1 \AA$ ), we were able to complete Nic96 by including helix $\alpha 9$, and two loops including 30 additional residues. We speculate that the improvement of the data is due to the ability of the nanobody to stabilize Nic96 in one conformation. Either this is an effect of the nanobody alone, or it may be a combination of nanobodybinding paired with crystal packing.

Several NPC nanobodies localize to the NE in vivo. Having characterized this nanobody library in vitro, we asked whether these nanobodies would bind their targets in vivo and whether their expression would affect cellular fitness. We first put the 
a

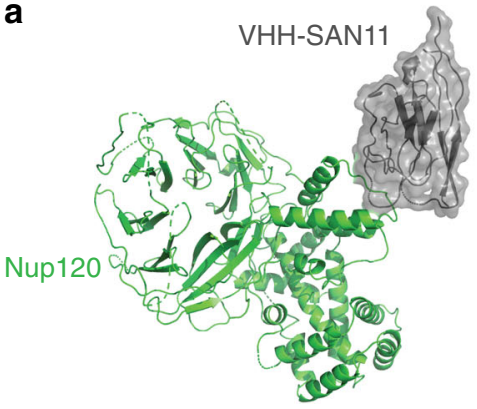

Ligand:

VHH-SAN10

VHH-SAN11

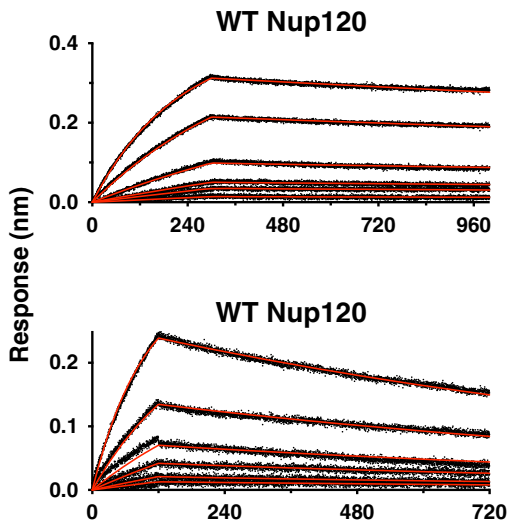

b
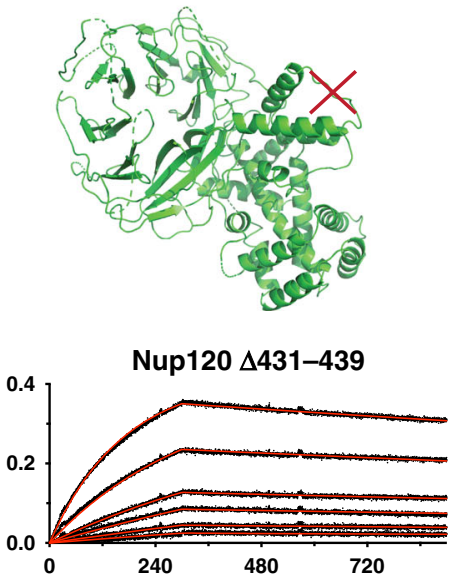

Nup120 $\Delta 431-439$

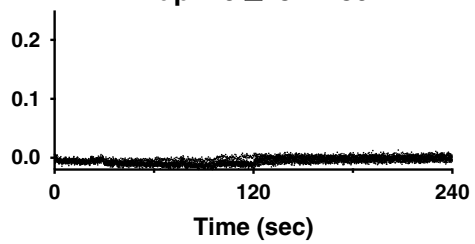

c

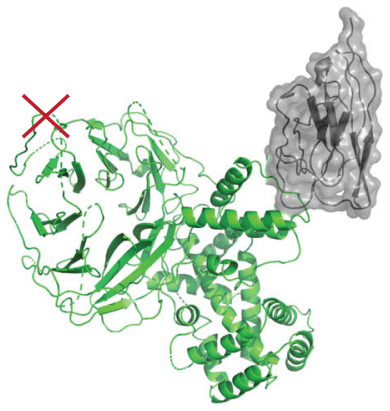

Nup120 $\Delta 187-203$

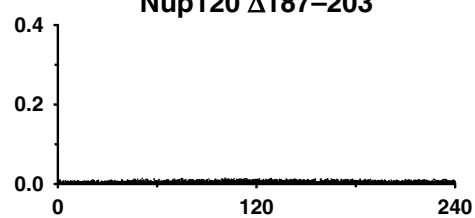

Nup120 $\Delta 187-203$

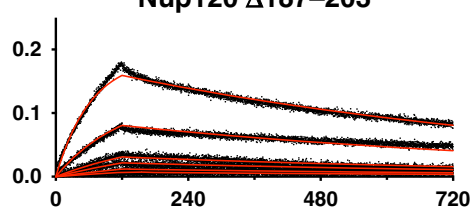

Fig. 4 Mutational analysis of Nup120 confirms the binding site of VHH-SAN10. Bio-layer interferometry (BLI) showing association and dissociation kinetics for VHH-SAN10 and VHH-SAN11 with Nup120 mutants. Nanobodies with a biotinylated C-terminal Avi-tag were fixed (ligand) and nups used as analytes. Curves were corrected for buffer background. Each set of curves is a twofold dilution series from $10 \mathrm{nM}$ analyte. Data are indicated by the black dotted lines and the red lines show the globally fitted curves. a Structure of Nup1201-757-VHH-SAN11 and BLI data of wild type (WT) Nup120 $1-757$ as the

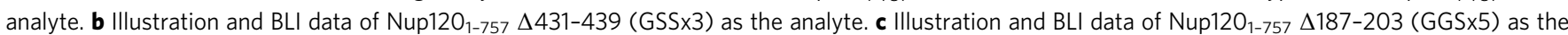
analyte.

production of each nanobody under control of the high expression $G A L$ promoter in a wild type yeast strain. We observed no fitness defects upon nanobody expression at both $30^{\circ} \mathrm{C}$ and $37^{\circ} \mathrm{C}$ (Fig. 6a). Given the small size of a nanobody relative to the NPC, this was not entirely surprising, but also suggests that none of the surfaces occupied by the nanobodies are essential for NPC integrity, assuming that the nanobodies can find their targets in the cell. We therefore asked whether the nanobodies localized to the NPC and the NE in vivo, thus examining their ability to bind their targets within the context of the assembled NPC. To this end, we fused mKate2, a monomeric far-red fluorescent protein ${ }^{40}$, to the $\mathrm{C}$ terminus of each nanobody. We then expressed these nanobody-mKate2 fusions in a yeast strain with endogenously GFP-tagged Nup120 (Nup120-GFP) as a Y complex and NPC marker, and examined co-localization. The expression of the nanobody-mKate2 fusions had only minor defects on fitness at $37^{\circ} \mathrm{C}$, with the exception of VHH-SAN7, which recognizes Sec13 (Fig. 6a). As none of the nanobody-mKate2 fusions were lethal, we converted the GAL promoter to the Nup120 promoter. We opted to perform the localization analysis using the weaker Nup120 promoter to maintain a similar stoichiometry of the nanobody to NPC components ${ }^{28}$ rather than to vastly overexpress the nanobody or have any confounding effects from GAL induction.

In the absence of any nanobody expression, we observed clear nuclear rim fluorescence for Nup120-GFP, while mKate2 alone produced diffuse fluorescence throughout the cytoplasm and the nucleus (Fig. 6b, 'Mock'). Of the three Nup85 nanobodies, one exhibited strong co-localization with Nup120 at the nuclear rim (VHH-SAN3) while the other two (VHH-SAN1 and 2) displayed reduced rim colocalization. VHH-SAN4 and 5 nanobodies, which recognize Nup133 at its $\mathrm{N}$-terminal $\beta$-propeller, did not colocalize with Nup120 but instead were distributed diffusely throughout the cell. VHH-SAN6, which recognizes Nup145C in the Y complex hub, also strongly enriched with Nup120 at the nuclear rim. VHH-SAN7 formed puncta in the cytoplasm, possibly due to binding Sec13 in the COPII vesicle coat or SEA complex, rather than forming a complex with the copies of Sec13 in the NPC ${ }^{47-49}$. We suggest that binding of VHH-SAN7 to these cytoplasmic copies of Sec13 may also account for the fitness defect of this strain. The majority of cells expressing both Nup84 nanobodies (VHH-SAN8 and 9), also showed strong nuclear rim localization with Nup120. However, VHH-SAN9 had a curious effect on Nup120 localization. While some cells expressing VHH-SAN9 showed strong colocalization with Nup120-GFP, many other cells had no or diffuse localization of Nup120-GFP on the NE to which VHH-SAN9 was localized. For the Nup120 nanobodies, both VHH-SAN11 and 12 showed strong localization at the nuclear rim. Finally, VHH-SAN12, which recognizes Nic96, presented as foci at the nuclear rim. Taken together, we find that the expression of our nanobodies in S. cerevisiae does not obviously affect cellular fitness. Many of these nanobodies localize to the NPC in vivo, confirming their potential as tools for future experiments in understanding NPC composition and assembly.

\section{Discussion}

Here we describe a 12-member library composed of nanobodies that bind both the Y complex and Nic96. The nanobodies bind their targets tightly, with all but one nanobody having an 
a

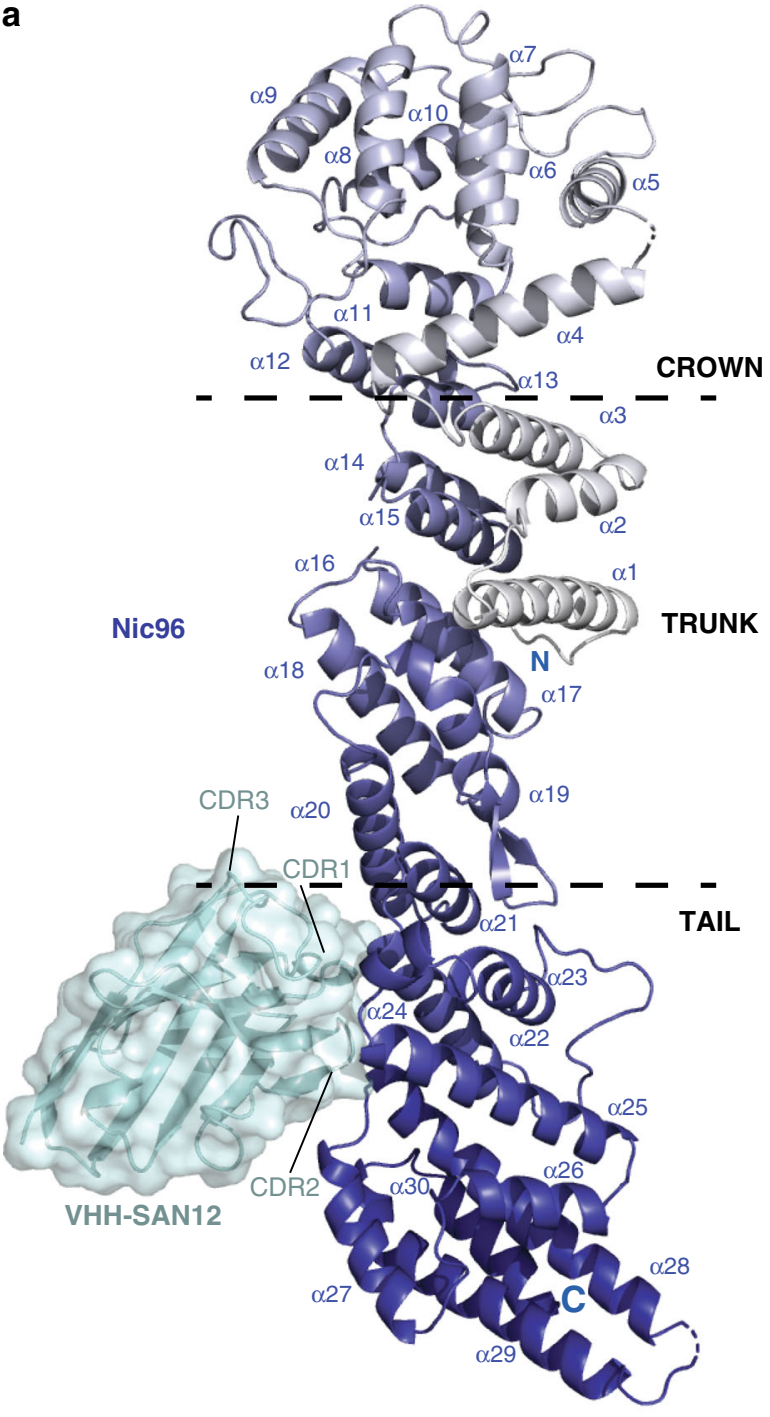

b

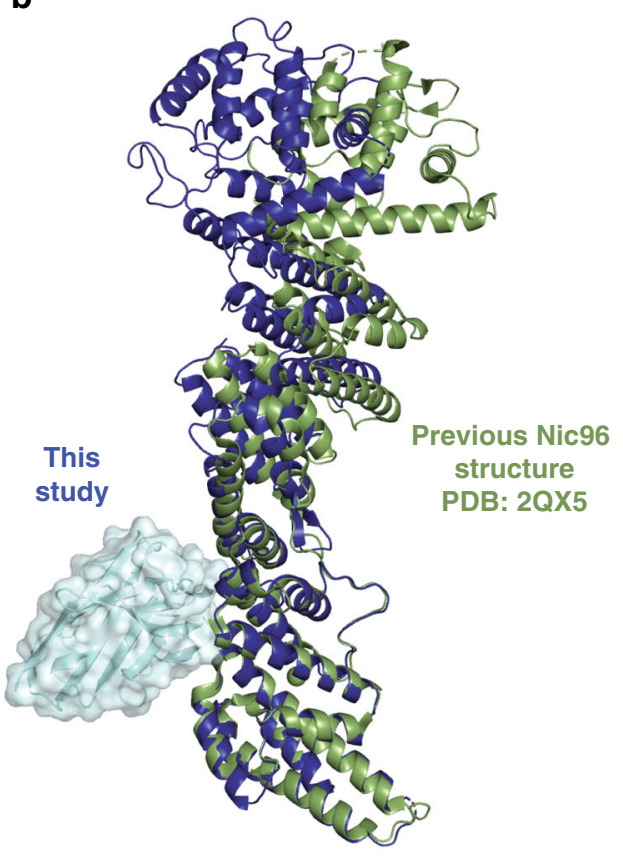

C

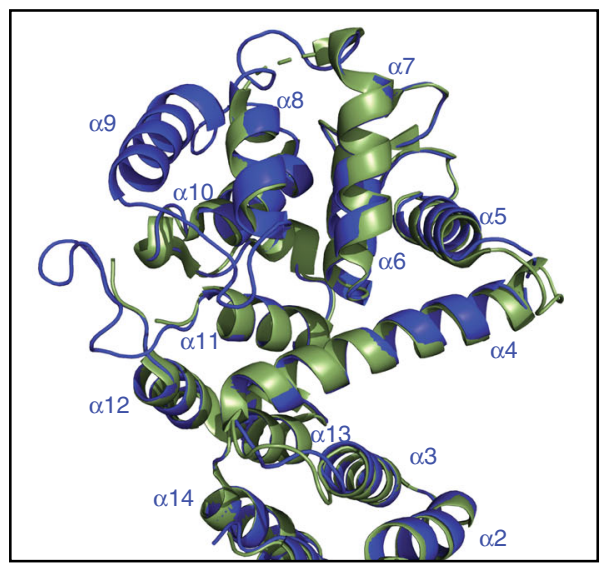

Fig. 5 Structure of Nic96-VHH-SAN12 highlights the flexibility of the ACE1 fold. a The structure of Nic96-VHH-SAN12. Nic96 is shown in gradient color from white to dark blue and VHH-SAN12 is shown in light blue. Helices are labeled on Nic96, along with the complementarity determining region (CDR) loops on VHH-SAN12. Boundaries of the ancestral cotamer element (ACE1) fold modules are indicated by the dashed lines. b Superposition of Nic96 with the previously solved structure (PDB: $2 \mathrm{QX} 5^{37}$ ) (shown in green). The alignment was done only in the tail module of the protein. c Superposition of the Nic96 crown module between the two structures. This structure of Nic96, shown in dark blue and the previous structure, shown in green.

equilibrium binding constant of $<10 \mathrm{nM}$. Through both SEC and $\mathrm{X}$-ray crystallography, we mapped their general binding sites or epitopes. The recognized epitopes range from relatively unstructured loops to clefts between domain interfaces. We also expressed these nanobodies in yeast cells to assess their ability to localize to NPCs in their native cellular environment. Armed with this information, we can now interpret the heterogeneity of the observed in vivo effects and identify elements of this library that can aid in future NPC assembly studies.

The Nup85 crown is thought to be adjacent to the Nup82 complex, where both VHH-SAN2 and 3 bind $^{24,30}$ (Fig. 7a, b). This correlates well with the localization of VHH-SAN2 being largely diffuse throughout the cell, suggesting that the $\mathrm{VHH}-$ SAN2 binding site is occluded. However, there is still some enrichment at the NE with Nup120, suggesting the Nup82-Nup85 tether may be flexible or dynamic, allowing for a fraction of the nanobody pool to still bind. Another possibility is that some Nup85 epitopes may be accessible, while others are not. This would suggest that Nup85 is differently assembled in different parts of the NPC. Both VHH-SAN1 and VHH-SAN3 are strongly enriched at the NE. However, the expression of VHH-SAN1 also yielded the unexpected formation of VHH-SAN1-Nup120 puncta away from the NE. We hypothesize that this nanobody may weaken the affinity of the Y complex to the NPC assembly. These puncta could represent $\mathrm{Y}$ complexes that are slower to incorporate into the NPC assembly or potentially dissociated Y complexes from assembled NPCs. In either case, this is only a modest disruption, as the cells have no obvious growth defect.

Both Nup133 nanobodies (VHH-SAN4 and 5) were unable to localize to the NE in yeast cells (Fig. 6b). These nanobodies are most likely blocked from binding in the assembly by the ArfGAP1 lipid packing sensing (ALPS) motif ${ }^{50}$ (Fig. 7b, d). Nup133 is thought to be anchored to the NE by its ALPS motif on its Nterminal $\beta$-propeller domain ${ }^{51,52}$, which is on the same face of Nup133 as the epitopes for VHH-SAN4 and $5^{20}$. This suggests that the ALPS interaction with the membrane outcompetes the 
a

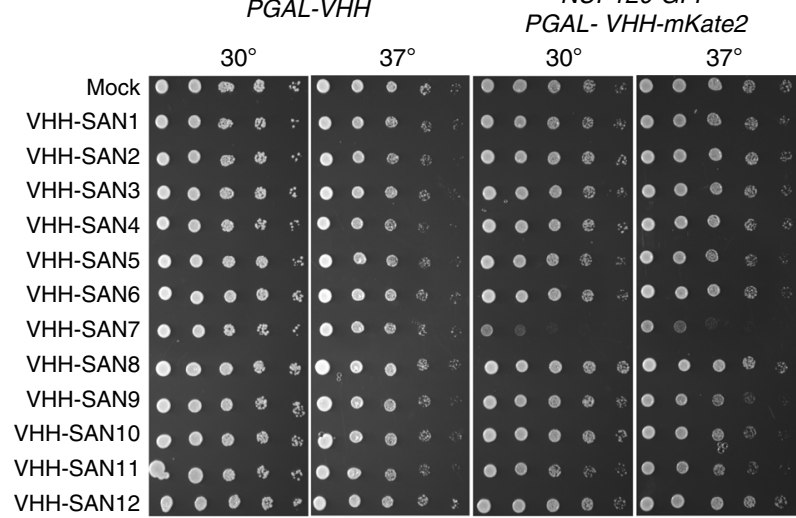

b

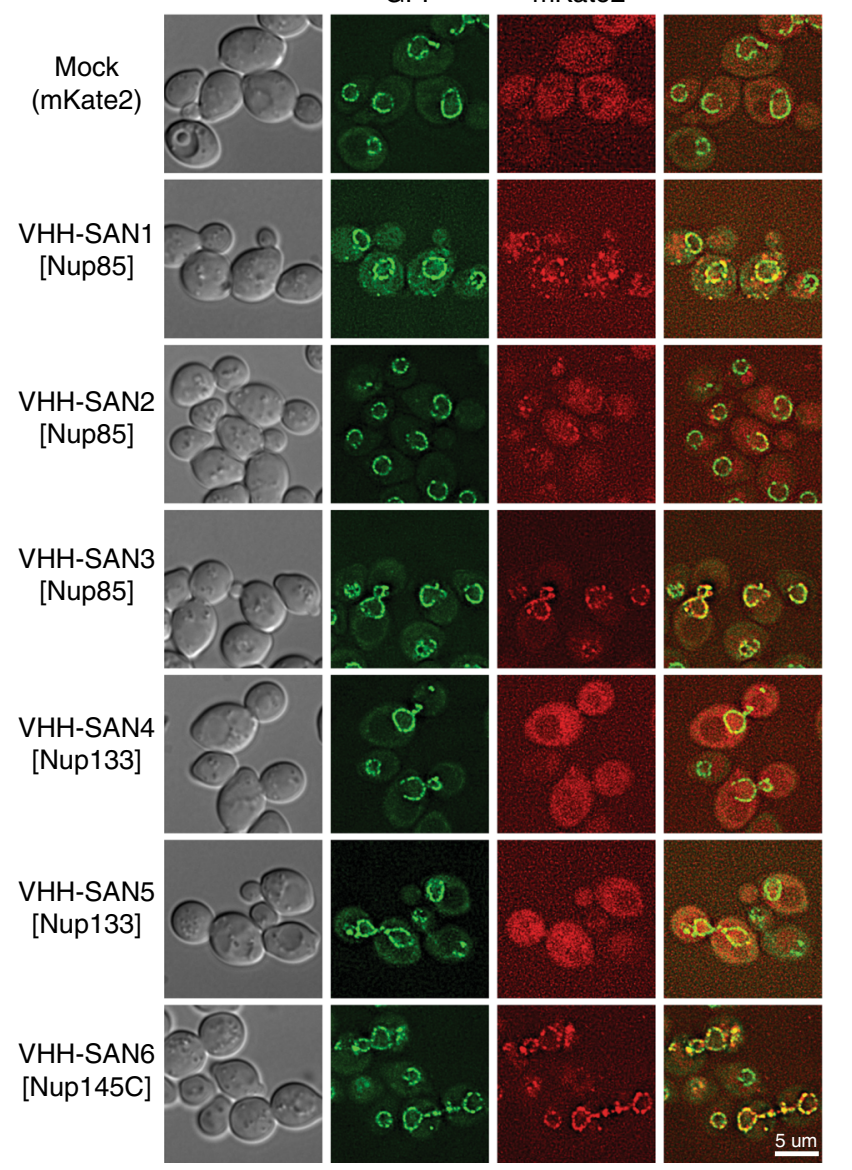

C

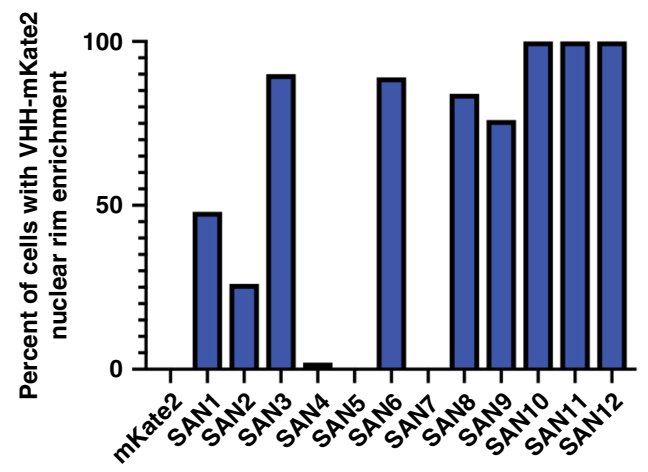

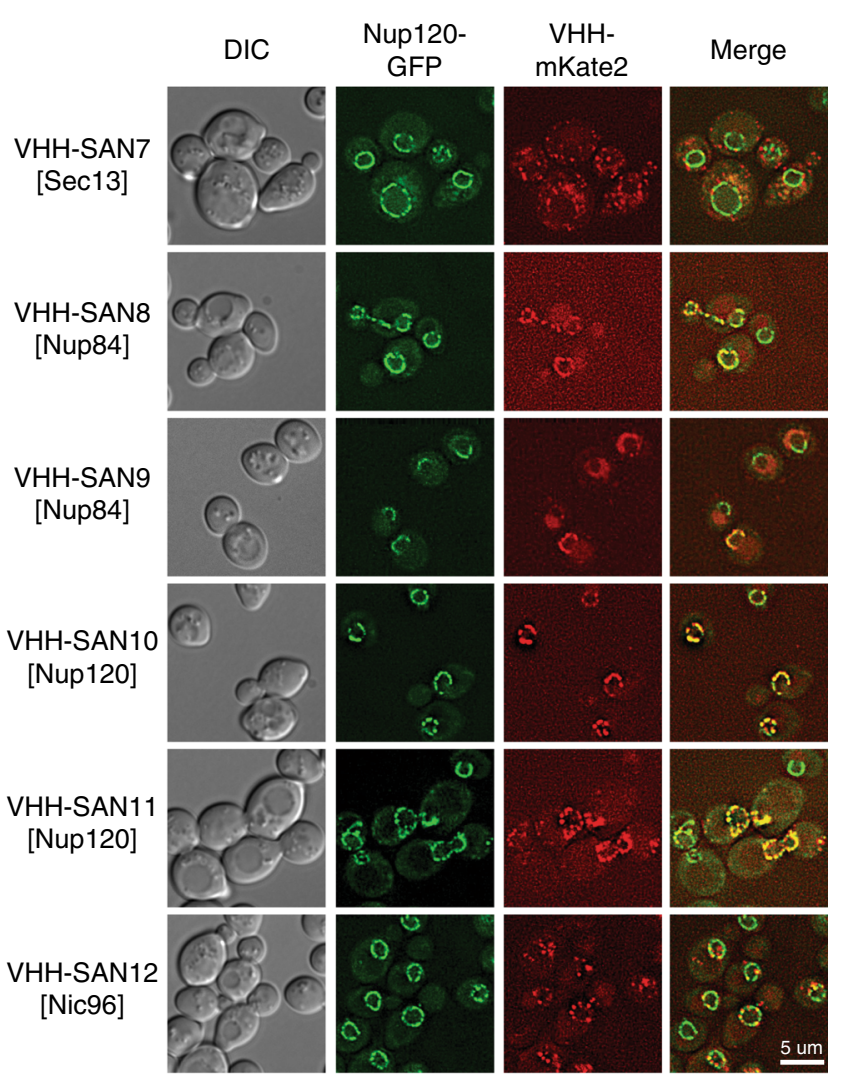

Fig. 6 Expression of nanobody-mKate2 fusions in vivo. a Spot assays for nanobody and nanobody-mKate2 expression. Strain construction is indicated at the top of the figure, along with assay temperature. Pictures were taken after $48 \mathrm{~h}$ on $2 \%$ galactose containing agar media. Mock specifies mKate 2 expression alone. b Fluorescence images of cells expressing endogenously tagged Nup120-GFP and plasmid expressed NUP120-VHH-mKate2 in DIC, GFP, and far-red channels with a merged overlay. Target of each nanobody is listed in brackets. Scale bar: $5 \mu \mathrm{M}$. c Percent of cells with VHH-mKate2 exhibiting nuclear rim enrichment, $n=50$ cells per strain over one experiment.

binding of the nanobodies. While VHH-SAN4 has both fast on and off kinetics and the weakest binding affinity $(230 \mathrm{nM})$, VHH-SAN5 binds Nup133 tightly in vitro (10 nM). This implies that the membrane interaction of the ALPS motif must have an even higher affinity or the epitope for VHH-SAN4 and 5 on Nup133 is blocked by the membrane very soon after new copies of Nup133 are synthesized, since the nanobodies are constitutively expressed.
Similar to VHH-SAN1, the Nup145C-specific VHH-SAN6 redistributes some Nup120 (and potentially Y complex) into cytoplasmic puncta, but does so to a lesser extent, along with its colocalization on the NE (Fig. 6b). Like VHH-SAN1 expression, the presence of these puncta has no effect on the fitness of the strain, so the pool of Y complex present on the NE must still be sufficient for proper cellular function. We cannot exclude at present that the expression of nanobodies like VHH-SAN1 might 


\section{Cytoplasmic ring}

a

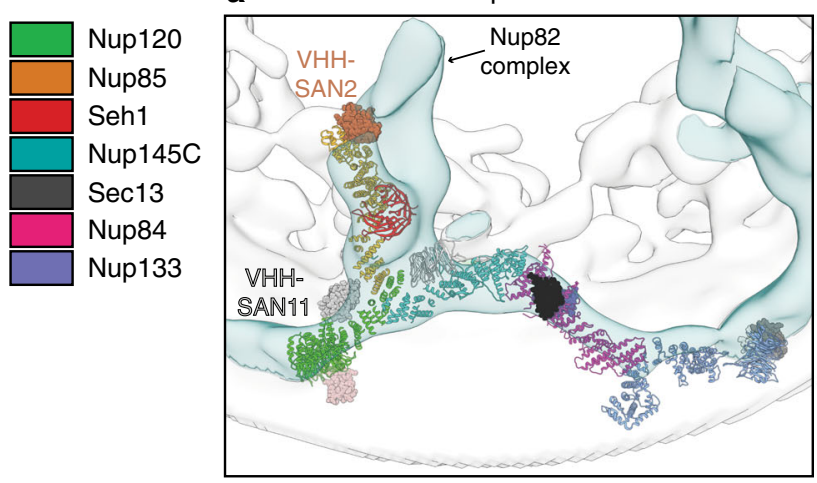

b

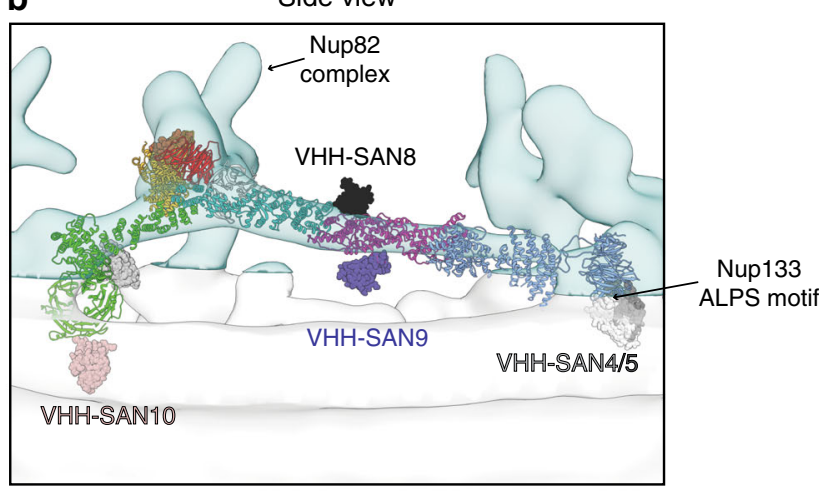

Nucleoplasmic ring

C

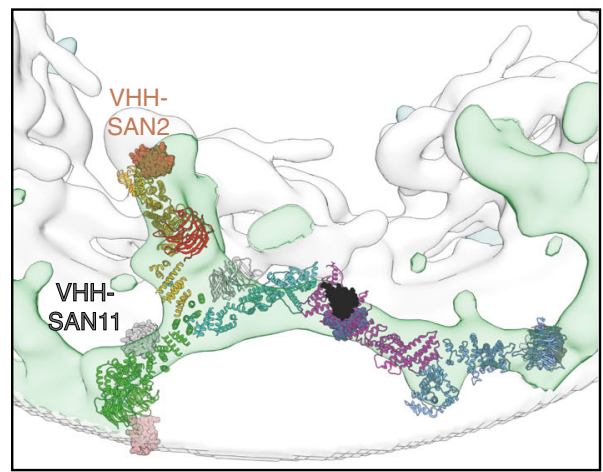

d

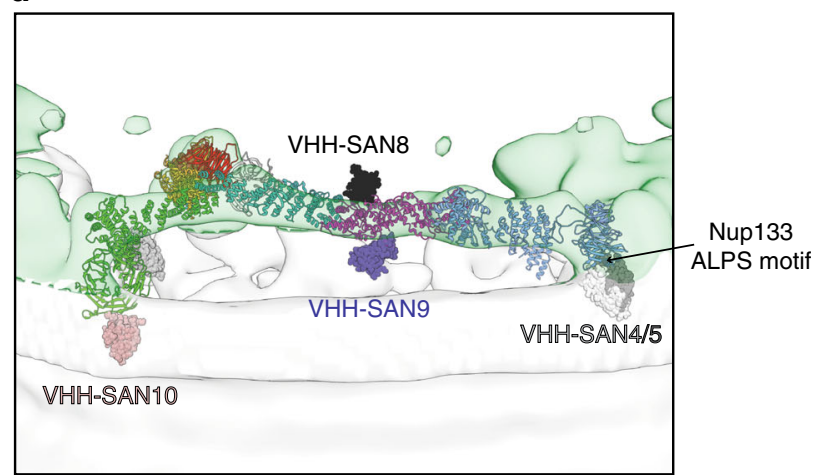

Fig. 7 Docking of the $\mathbf{Y}$ complex and nanobodies into the cryo-ET map of the NPC. a Top and $\mathbf{b}$ side view of the cryo-ET map from S. cerevisiae (EMD10198) with the density for corresponding to the cytoplasmic ring of the NPC colored in light blue. A top-scoring global fit for the composite S. cerevisiae $Y$ complex model shown in both a top and side view. Y complex nups colored as indicated in the legend (right). Nanobodies were not included during global fitting, nanobody-nup complexes were superposed after docking. Nanobodies are shown with surface rendering and labeled with matching colors. Density corresponding to the Nup82 complex is indicated. The position of the Nup133 ALPS motif adjacent to the nuclear envelope is also indicated. c Top and d side view cryo-ET map with the density corresponding to the nucleoplasmic ring of the NPC colored in light green. Top-scoring global fit for the composite S. cerevisiae $Y$ complex model shown in both a top and side view. Nanobody positioning, coloring, and labeling as in $\mathbf{a}$, $\mathbf{b}$.

exert more subtle effects, for example by affecting the extent or rate of nuclear import/export of select cargoes. The Sec13 nanobody, VHH-SAN7, is the only nanobody in the library that decreases fitness as a mKate 2 fusion, but not when expressed alone (Fig. 6a). From our fluorescence localization data, VHHSAN7 exists mostly in cytoplasmic puncta, potentially due to the fact that Sec13 is present not only in the NPC, but also the COPII vesicle coat and SEA complex ${ }^{47,48}$. We hypothesize that $\mathrm{VHH}-$ SAN7 binds the copies of Sec13 outside of the NPC present in the cytoplasm, possibly by interacting with these copies of Sec13 more quickly after translation. Although we know VHH-SAN7 can bind Sec13 when assembled into the Y complex hub in vitro, it is also possible that its binding site is occluded in the context of the assembled NPC (Supplementary Fig. 1).

The Nup84 specific nanobodies (VHH-SAN8 and 9) behave differently due to binding opposite faces of Nup84. VHH-SAN8 binds the 'top surface of Nup84, away from the NE (Fig. 7b, d). This most likely explains why VHH-SAN8 colocalizes strongly with Nup120-GFP and causes no fitness defects. On the other hand, the expression of VHH-SAN9 has a peculiar effect on the localization of Nup120-GFP. Many cells showed diffuse fluorescence for VHH-SAN9, but in the general curvature of the NE. In many cases, we observed similar diffuse NE fluorescence of Nup120-GFP on part of the NE, but crisp NE rim fluorescence on the part of the nucleus where VHH-SAN9 was not present. VHH-
SAN9 binds the side of Nup84 adjacent to the NE (Fig. 7b, d). It is possible that the presence of VHH-SAN9 disrupts the Y complex assembly on the NE, prying the $\mathrm{Y}$ complex away from the membrane. Interestingly, this occurs on only some of the NPCs within the same cell. Further investigation into the state of these NPCs and the NE is ongoing.

Both Nup120 specific nanobodies colocalize well with Nup120GFP. The expression of VHH-SAN10 has little effect on the distribution of rim fluorescence of Nup120-GFP. Residues 197-216 are hypothesized to also be an ALPS motif and the overlapping residues 187-203 are required for VHH-SAN10 binding ${ }^{52}$. Docking of the Y complex into the cryo-ET map of the scNPC suggests this face of Nup120 to be positioned adjacent to the membrane ${ }^{24}$ (Fig. 7b, d). If Nup120 does bind the membrane at this loop, the nanobody likely outcompetes the affinity of Nup120 for the membrane. This would also suggest that the membrane-attachment by Nup120 may not be critical for NPC assembly and function. VHH-SAN11 had a more pronounced effect on the localization of Nup120-GFP. Some protrusions emanating from the NE were observed to have both Nup120 and VHH-SAN11, along with some foci in the cytoplasm.

VHH-SAN12 binds Nic96 between its trunk and tail modules. The interaction between the CDR loops of VHH-SAN12 and these trunk-tail interface helices maintains Nic96 in a different conformation than previously observed by X-ray crystallography 45,46 
Most of the VHH-SAN12-mKate2 fusion enriched at the NE along with Nup120-GFP. However, VHH-SAN12 formed foci, both on the NE and in the cytoplasm and within the nucleus, while Nup120-GFP showed an even distribution on the NE. There are two possible explanations that can account for these foci. Either VHH-SAN12-mKate2 expression was limiting or not every NPC displays Nic96 in a conformation or configuration accessible for VHH-SAN12 binding. The concept of NPC heterogeneity has been observed and discussed in the literature, both in terms of its composition and in size ${ }^{53-55}$. Whether this interesting fluorescence is indeed due to NPC heterogeneity within the same cell is also under ongoing investigation.

The nanobody suite describes here provides a set of tools for studying the NPC assembly both in vitro and in vivo. In vitro, the nanobodies bind tightly to their targets and have enabled structural analysis of multiple nups at higher resolution than reported earlier or that evaded previous attempts by X-ray crystallography altogether ${ }^{20}$. In vivo, many nanobodies colocalize with the $\mathrm{Y}$ complex and therefore can be used as cellular tools in future studies on the NPC. For example, the nanobody library could enable subunit identification in future cryo-ET studies. In several cases, such as VHH-SAN9 and VHH-SAN12, they have also provided questions for further study on NPC assembly and heterogeneity. Along with ref. ${ }^{20}$, we also detailed the library's breadth in antigen recognition, owing to its wide diversity in both CDR sequence and length. Overall, our data highlight the exciting potential of this nanobody library to be used as tools for both in vitro and in vivo studies of the NPC and pave the way for future explorations of NPC assembly and composition in $S$. cerevisiae.

\section{Methods}

Construct generation. All Nups (Nup120, Nup85, Seh1, Nup145C, Sec13, Nup84, Nup133, Nic96) were cloned from S. cerevisiae and expressed recombinantly in E. coli. Expression constructs for His-tagged Nup84, Nup85, Nup85 $200-383$ (Nup85 $5_{\text {crown }}$ ), Nup85 ${ }_{1-564}$-Seh1 (Nup85 crown-trunk $^{-S e h 1), ~ N u p 120}{ }_{855-1037^{-}}$ Nup145C H4-712-Sec13, Nup120 $_{1-757}$, Nup120-Nup145C-Sec13, Sec13-

Nup145C $\mathrm{C}_{320-411}\left(\mathrm{Sec} 13-\mathrm{Nup} 145 \mathrm{C}_{\text {blade }}\right)$, and Nic96 $6_{186-839}$ were published previously $18,35,40,45,56$. In addition, we used N-terminally fused 14xHis, bdSUMO tagged full-length Nup133 and N-terminally fused 6xHis tagged Nup133 $55-481$ (Nup133 $3_{\text {NTD }}$ ).

Upon VHH selection by phage display and ELISA, each VHH was sub-cloned for expression. Each $\mathrm{VHH}$ sequence was N-terminally fused with a $14 \mathrm{xHis}$ bdSUMO $\operatorname{tag}^{57}$ and cloned into a T7-promoter-based bacterial expression vector with ampicillin resistance. A separate construct for each $\mathrm{VHH}$ was created with a C-terminally fused Avi-tag ${ }^{36}$ for biotinylation. Primers used for cloning are listed in Supplementary Table 1.

Protein expression and purification. Nup120-Nup145 $\mathrm{C}_{34-712}-\mathrm{Sec} 13$, Nup85Seh1, Nup120 ${ }_{855-1037-N u p 145 C_{34-712}-\text { Sec13, Nup84, Nic96 }}$ 189-839, Nup133 $_{\text {NTD }}$, Sec13-Nup145C blade, Nup85 $5_{\text {crown-trunk }}$-Seh1, and Nup85 $5_{\text {crown }}$ expression vectors were transformed into E. coli LOBSTR-RIL(DE3) ${ }^{58}$ (Kerafast) cells and protein production was induced with $0.2 \mathrm{mM}$ IPTG at $18^{\circ} \mathrm{C}$ for $12-14 \mathrm{~h}$. Cells were collected by centrifugation at $6000 \times g$, resuspended in lysis buffer $(50 \mathrm{mM}$ potassium phosphate $\mathrm{pH} 8.0,500 \mathrm{mM} \mathrm{NaCl}, 30-40 \mathrm{mM}$ imidazole, $3 \mathrm{mM} \beta$-mercaptoethanol $(\beta M E), 1 \mathrm{mM}$ PMSF) and lysed using a high-pressure cell homogenizer (Microfluidics LM20). The lysate was cleared by centrifugation at $12,500 \times g$ for $25 \mathrm{~min}$. The soluble fraction was incubated with Ni Sepharose 6 Fast Flow beads (GE Healthcare) for $30 \mathrm{~min}$ on ice. After washing the beads with lysis buffer, the protein was eluted (250 mM imidazole $\mathrm{pH} 8.0,150 \mathrm{mM} \mathrm{NaCl}, 3 \mathrm{mM} \beta \mathrm{ME}$ ).

All VHH constructs and Nup133 were transformed, grown, harvested, and lysed as above. For Avi-tagged VHH constructs, $20 \mathrm{mM}$ biotin was added to the cultures prior to IPTG induction. After lysis, the soluble fraction was incubated with Ni Sepharose 6 Fast Flow beads (GE Healthcare) for 30 min on ice. The beads were then washed with lysis buffer and transferred to low imidazole buffer $(50 \mathrm{mM}$ potassium phosphate $\mathrm{pH} 8.0,500 \mathrm{mM} \mathrm{NaCl}, 10 \mathrm{mM}$ imidazole, $3 \mathrm{mM} \beta \mathrm{ME}$ ) along with $10 \mu \mathrm{g}$ SEN-p protease and incubated $2 \mathrm{~h}$ at $4{ }^{\circ} \mathrm{C}$. The flow through containing the cleaved protein was collected, along with a 2 -column volume $(\mathrm{CV})$ wash with low imidazole buffer. Cut tags and uncut protein was eluted as above.

Buffers used in further purification are as follows: gelfiltration (GF) buffer ( $150 \mathrm{mM} \mathrm{NaCl}, 10 \mathrm{mM}$ Tris/HCl pH 7.5, $1 \mathrm{mM}$ DTT, $0.1 \mathrm{mM}$ EDTA), Dialysis buffer (100 mM NaCl, $10 \mathrm{mM}$ Tris/HCl pH 7.5, $1 \mathrm{mM}$ DTT, $0.1 \mathrm{mM}$ EDTA), $\mathrm{S}$ buffers (0 or $1 \mathrm{M} \mathrm{NaCl}, 10 \mathrm{mM}$ Tris/ $\mathrm{HCl} \mathrm{pH} 7.5,1 \mathrm{mM}$ DTT, $0.1 \mathrm{mM}$ EDTA) and Q buffers (0 or $1 \mathrm{M} \mathrm{NaCl}, 20 \mathrm{mM}$ HEPES/NaOH pH 8.0, $1 \mathrm{mM}$ DTT, $0.1 \mathrm{mM}$ EDTA).

After Ni purification, Nup120-Nup145C ${ }_{34-712}-\mathrm{Sec} 13$, Nup120 ${ }_{855-1037^{-}}$ Nup145C 34-712-Sec13, Nup85-Seh1, Nup84, Nic96 $_{189-839}$, Nup133 ${ }_{\text {NTD }}$, Sec13Nup145C blade, Nup85 crown-trunk $_{\text {-Seh1, Nup120 }}$ 1-757, and Nup133 were incubated with 3C protease and dialyzed into dialysis buffer overnight. Nup120Nup145C $34-712-$ Sec13, Nup84, Nup85 $5_{\text {crown }}$, and Nup120 $1-757$ were run over a HiTrap SP FF column (GE Healthcare), collecting the flow through. Nup133 was loaded onto a MonoQ column (GE Healthcare) and eluted over a gradient of

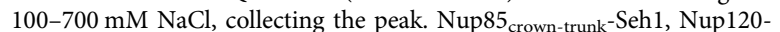
Nup145C 34-712-Sec13, Nup85-Seh1, Nup84, Nic96 $_{189-839}$, and Nup133 were concentrated and loaded onto a pre-equilibrated Superdex S200 16/60 column (GE

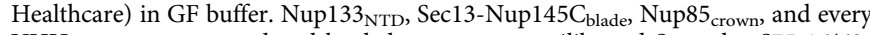
VHH were concentrated and loaded onto a pre-equilibrated Superdex S75 16/60 column (GE Healthcare) in GF buffer. All fractions were analyzed by SDS-PAGE, peaks were pooled and concentrated.

For all Nup-VHH complexes, the proteins were incubated for $30 \mathrm{~min}$ on ice. The incubated mixtures were then run over a Superdex S75 or S200 10/300 column pre-equilibrated in GF buffer. Fractions containing the complexes were pooled and concentrated. To assemble the Y complex, we first mix 1.5× molar excess of Nup85-Seh1 with Nup120-Nup145C $34-712-S e c 13$. After incubation for $30 \mathrm{~min}$ on ice, the complex was run over a Superdex S200 10/300 column in GF buffer. Fractions containing all five proteins were pooled and concentrated. We also mixed $1.5 \times$ molar excess of Nup84 with Nup133 and followed the same protocol. These two complexes were then mixed together with $1.5 \times$ molar excess of Nup84-Nup133 and run over a Sepharose 6 10/300 column (GE Healthcare). Fractions containing all Y complex components were pooled and concentrated.

VHH library and M13 phage generation. Alpaca immunization and library generation were done as previously described ${ }^{59}$. The animal was purchased locally, maintained in the pasture, and immunized following a protocol authorized by the Tufts University Cummings Veterinary School Institutional Animal Care and Use Committee (IACUC). The animal was immunized against recombinantly expressed full-length Y complex (Nup120-Nup85-Seh1-Nup145C-Sec13-Nup84-Nup133). The library was then grown to mid-log phase in $100 \mathrm{ml} \mathrm{SOC}$ with $50 \mu \mathrm{g} / \mathrm{ml}$ ampicillin. Then, the culture was infected with $100 \mu \mathrm{l} 10^{14} \mathrm{PFU} / \mathrm{ml}$ VCSM13 helper phage. Following $2 \mathrm{~h}$ incubation at $37^{\circ} \mathrm{C}$, the cells were harvested by centrifugation and re-suspended in $100 \mathrm{ml} 2 \mathrm{YT}, 0.1 \%$ glucose, $50 \mu \mathrm{g} / \mathrm{ml} \mathrm{kanamycin}$, and $50 \mu \mathrm{g} / \mathrm{ml}$ ampicillin. Cultures were incubated overnight at $30^{\circ} \mathrm{C}$, then centrifuged for $20 \mathrm{~min}$ at $7700 \times g$, followed by phage precipitation from the resulting supernatant with $1 \%$ PEG-6000, $500 \mathrm{mM} \mathrm{NaCl}$ at $4^{\circ} \mathrm{C}$, and resuspended in PBS.

Selection of VHHs by phage display. VHHs were selected by panning against Nup120 ${ }_{855-1037-N u p 145 C_{34-712}-\text { Sec13, Nup85-Seh1, Nic96 }}{ }_{189-839}$, Nup84, and Nup133 $35-481$. Hundred micrograms of recombinant protein was biotinylated by coupling Chromalink NHS-biotin reagent (Solulink) to primary amines for $90 \mathrm{~min}$ in $100 \mathrm{mM}$ sodium phosphate $\mathrm{pH} 7.4,150 \mathrm{mM} \mathrm{NaCl}$. Unreacted biotin was removed using a Zeba desalting column (Thermo Fisher). Biotin incorporation was monitored using absorbance at $354 \mathrm{nM} .100 \mu \mathrm{l}$ MyOne Streptavidin-T1 Dynabeads (Life Technologies) were blocked in $2 \%(\mathrm{w} / \mathrm{v})$ bovine serum albumin (Sigma) in PBS for $2 \mathrm{~h}$ at $37^{\circ} \mathrm{C}$. Twenty micrograms of biotinlyated antigen in PBS was added to the blocked beads and incubated for $30 \mathrm{~min}$ at $25^{\circ} \mathrm{C}$ with agitation. The beads were then washed three times in PBS and $200 \mu \mathrm{l}$ of $10^{14} \mathrm{PFU} / \mathrm{ml} \mathrm{M} 13$ phage displaying the VHH library were added in $2 \%$ BSA in PBS for $1 \mathrm{~h}$ at room temperature. The beads were then washed 15 times with PBS, $0.1 \%$ Tween-20 (PBST). Phage was eluted by the addition of E. coli ER2738 (NEB) for $15 \mathrm{~min}$ at $37^{\circ} \mathrm{C}$, followed by elution with $200 \mathrm{mM}$ glycine, $\mathrm{pH} 2.2$, for $10 \mathrm{~min}$ at $25^{\circ} \mathrm{C}$. The eluate was neutralized with $1 \mathrm{M}$ Tris/ $\mathrm{HCl} \mathrm{pH} 9.1$, pooled with the $E$. coli culture, and plated onto $2 \mathrm{YT}$ agar plates supplemented with $2 \%$ glucose, $5 \mu \mathrm{g} / \mathrm{ml}$ tetracycline, and $10 \mu \mathrm{g} / \mathrm{ml}$ ampicillin, and grown overnight at $37^{\circ} \mathrm{C}$. A second round of panning was performed with the following modifications: $2 \mu \mathrm{g}$ of biotinylated antigen was used as bait, and incubated with $2 \mu 110^{14} \mathrm{PFU} / \mathrm{ml} \mathrm{M} 13$ phage displaying the firstround VHH library for $15 \mathrm{~min}$ at $37^{\circ} \mathrm{C}$, followed by 15 washes in PBST.

ELISA. Following two rounds of phage panning, 96 colonies were isolated in 96well round-bottom plates and grown to mid-log phase at $37^{\circ} \mathrm{C}$ in $200 \mu \mathrm{l} 2 \mathrm{YT}, 10$ $\mu \mathrm{g} / \mathrm{ml}$ ampicillin, $5 \mu \mathrm{g} / \mathrm{ml}$ tetracycline, induced with $3 \mathrm{mM}$ IPTG and grown overnight at $30^{\circ} \mathrm{C}$. Plates were centrifuged at $12,000 \times g$ for $10 \mathrm{~min}$, and $100 \mu \mathrm{l}$ of supernatant was mixed with an equal volume of $5 \%(\mathrm{w} / \mathrm{v})$ nonfat dry milk in PBS. This mixture was added to an ELISA plate coated with $1 \mu \mathrm{g} / \mathrm{ml}$ antigen. Following four washes with $1 \%$ Tween-20 in PBS, anti-llama-HRP antibody (Bethyl) was added at a 1:10,000 dilution in 5\% (w/v) nonfat dry milk in PBS for $1 \mathrm{~h}$ at $25^{\circ} \mathrm{C}$. The plate was developed with fast kinetic TMB (Sigma) and quenched with $1 \mathrm{M}$ $\mathrm{HCl}$. Absorbance at $450 \mathrm{~nm}$ was determined in a plate reader (Spectramax; Molecular Devices).

Biolayer interferometry. Streptavidin biosensor tips were pre-incubated in BLI buffer (10 mM Tris/HCl pH 7.5, $150 \mathrm{mM} \mathrm{NaCl}, 1 \mathrm{mM}$ DTT, $0.1 \mathrm{mM}$ EDTA, 0.05\% Tween-20, $0.1 \%$ bovine serum albumin) for $10 \mathrm{~min}$, followed by immobilization of 
biotinylated, C-terminally Avi-tagged nanobody ligands to between $0.2-0.5 \mathrm{~nm}$ over 40-60 s. After dipping the coated biosensor tip in BLI buffer for $1 \mathrm{~min}$, association was measured in analyte over 1-80 min. Dissociation was measured in BLI buffer for 1-220 min. All binding sensorgrams were recorded on a forteBIO OctetRED96 instrument. All fits were done using global, 1:1 kinetic binding parameters using the Octet data analysis software.

Protein crystallization. Initial hits of Nic96 $6_{186-839}-\mathrm{VHH}-\mathrm{SAN} 12$ were obtained at $18{ }^{\circ} \mathrm{C}$ in 1 day in a 96 -well sitting drop tray with a reservoir containing $8 \%(\mathrm{w} / \mathrm{v})$ PEG 8,000 and 0.1 M tri-sodium citrate pH 5.0 (Protein Complex suite, Qiagen). Hanging drops of $1 \mu \mathrm{l}$ protein at $6 \mathrm{mg} / \mathrm{ml}$ and $1 \mu \mathrm{l}$ of precipitant (7-10\% (w/v) PEG 8000 and $0.1 \mathrm{M}$ tri-sodium citrate $\mathrm{pH} 5.0$ ) incubated at $18^{\circ} \mathrm{C}$ produced diffraction quality rod-shaped crystals in 3 days. Crystals were transferred into a cryoprotectant solution containing the crystallization condition with $15 \%$ (v/v) glycerol and cryo-cooled in liquid nitrogen.

Initial hits of Nup120 $1-757-\mathrm{VHH}-\mathrm{SAN} 10 / 11$ were obtained at $18^{\circ} \mathrm{C}$ in 3 days in a 96-well sitting drop tray with a reservoir containing $20 \%$ (w/v) PEG 8000, $0.2 \mathrm{M}$ magnesium chloride, $0.1 \mathrm{M}$ Tris/ $\mathrm{HCl} \mathrm{pH} 8.5$ (JCSG + suite, Qiagen). Hanging drops of $1 \mu \mathrm{l}$ protein at $2 \mathrm{mg} / \mathrm{ml}$ and $1 \mu \mathrm{l}$ of precipitant (19\% (w/v) PEG 8000, 0.1 $\mathrm{M}$ magnesium chloride, $0.1 \mathrm{M}$ Tris $/ \mathrm{HCl} \mathrm{pH} \mathrm{8.5)} \mathrm{incubated} \mathrm{at} 18^{\circ} \mathrm{C}$ yielded large rod-shaped crystals in 4 days. Crystals were transferred into a cryo-protectant solution containing the crystallization condition with $20 \%$ (v/v) glycerol and cryocooled in liquid nitrogen.

Initial hits of Nup85 ${ }_{1-564}$-Seh1-VHH-SAN2 were obtained at $18^{\circ} \mathrm{C}$ in 1 day in a 96-well sitting drop tray with a reservoir containing $1 \mathrm{M}$ ammonium sulfate and $0.1 \mathrm{M}$ sodium acetate $\mathrm{pH} 5.0$ (Protein Complex suite, Qiagen). Crystals significantly improved by changing the buffer to di-sodium succinate $\mathrm{pH} 5.5$ and the addition of $4 \%(\mathrm{v} / \mathrm{v}) 1$-propanol. Sitting drops of $0.2 \mu \mathrm{l}$ protein at $17 \mathrm{mg} / \mathrm{ml}$ and $0.2 \mu \mathrm{l}$ of precipitant yielded diffraction-quality, hexagonal rod-shaped crystals in 3 days that continued to grow over 3 weeks at $18^{\circ} \mathrm{C}$. Crystals were transferred into a cryo-protectant solution containing the crystallization condition with $15 \%(\mathrm{v} / \mathrm{v})$ PEG 200 and cryo-cooled in liquid nitrogen.

Structure determination. Data collection was performed at the advanced photon source end station 24-IDC. All data processing steps were carried out with programs provided through $\mathrm{SBgrid}^{60}$. Data reduction was performed using HKL $2000^{61}$. Statistical parameters of data collection and refinement are all given in Table 2. All manual model building steps were carried out with $\operatorname{Coot}^{62}$ and phenix. refine ${ }^{63}$ was used for iterative refinement. Structure figures were created in PyMOL (Schrödinger LLC).

The structure of Nic96 $186-839$-VHH-SAN12 was solved by MR using Phaser-MR in PHENIX. A two-part MR solution was obtained by sequentially searching with models of Nic96 and VHH-SAN12. For Nic96, we used the previously solved structure from S. cerevisiae (PDB:2QX5) ${ }^{45}$. For VHH-SAN12, we used a nanobody structure with its CDR loops removed (PDB:1BZQ) ${ }^{64}$. The asymmetric unit contains one copy of Nic96 $186-839^{-}$VHH-SAN12. Near the end of refinement, TLS refinement was used, with a significant impact on lowering the $\mathrm{R}$ factors.

The structure of Nup120 $1-757^{-}$VHH-SAN10/11 was solved by MR using PhaserMR in PHENIX, just as Nic96 $186-839-\mathrm{VHH}-\mathrm{SAN} 12$. We used the previously solved S. cerevisiae Nup120 $1-757$ structure as the search model (PDB:3HXR ${ }^{40}$. The asymmetric unit contains one copy of the complex. Many sidechains were removed from the model due to poor density. Secondary structure restrains were used throughout refinement. Regarding the two nanobodies, density only for $\mathrm{VHH}-$ SAN11 was present on the map.

The structure of Nup $85_{\text {crown-trunk }}$-Seh1-VHH-SAN2 was solved by MR using Phaser-MR in PHENIX. A solution was found by searching with the previously solved S. cerevisiae Nup85 ${ }_{1-564}$-Seh1 structure as the model (PDB:3EWE) ${ }^{18}$. The asymmetric unit contains one copy of the complex. Large difference density was present near the crown domain of Nup85, the known binding site of VHH-SAN2 biochemically (Supplementary Fig. 4), and we were able to manually place a model after refinement of Nup85 $5_{\text {crown-trunk }}$-Seh1. Since the CDR loops provide a significant amount of density nearest to Nup85, a model was generated by SWISSMODEL. Near the end of refinement, TLS parameters were used. For the final structure, occupancies for the VHH-SAN2 residues outside of the $2 \mathrm{Fo}-\mathrm{Fc}$ density at $1 \sigma$ were set to zero.

Yeast strain construction. The Nup120-GFP strain was made as previously described $^{40}$. In brief, C-terminal GFP-tagging was achieved by homologous recombination in a BY4741 background, using pFA6a-GFP(S65T)-kanMX6 as a template for C-terminal modifications ${ }^{65}$. Strains were selected on G418 plates (200 $\mu \mathrm{g} / \mathrm{ml}$ ) and verified by PCR. Nanobody expression for fluorescence experiments was done on a plasmid based on pAG416GAL-ccdB (Addgene). The plasmid was modified with the Nup120 promoter (500 bp upstream of the ORF) in place of the GAL promoter and each nanobody followed by a fused C-terminal mKate2 tag. Strains were selected and maintained by growth on SD-Ura media and verified by sequencing. Nanobody expression for toxicity experiments was done on a plasmid based on pAG416GAL-ccdB (Addgene). Each VHH sequence was inserted downstream of the GAL promoter and strains were selected and maintained by growth on SC-Ura media.
Table 2 Data collection and refinement statistics.

\begin{tabular}{|c|c|c|c|}
\hline Protein & $\begin{array}{l}\text { Nic96 }_{189-839 \prime} \\
\text { VHH-SAN12 }\end{array}$ & $\begin{array}{l}\text { Nup120 }_{1-757,} \\
\text { VHH-SAN10, } \\
\text { VHH-SAN11 }\end{array}$ & $\begin{array}{l}\text { Nup85, } \\
\text { Seh1, VHH- } \\
\text { SAN2 }\end{array}$ \\
\hline PDB code & $6 \times 07$ & $6 \times 06$ & $6 \times 08$ \\
\hline $\begin{array}{l}\text { Organism } \\
\text { Data collection }\end{array}$ & \multicolumn{3}{|c|}{ S. cerevisiae, V. pacos } \\
\hline \multicolumn{4}{|l|}{ Cell dimensions } \\
\hline$a, b, c(\AA)$ & $\begin{array}{l}48.1 \\
79.5,283.3\end{array}$ & $\begin{array}{l}\text { 193.2, } \\
\text { 193.2, } 78.1\end{array}$ & $\begin{array}{l}234.0 \\
234.0,139.4\end{array}$ \\
\hline$\alpha, \beta, \gamma\left(^{\circ}\right)$ & $90,90,90$ & $90,90,120$ & $90,90,120$ \\
\hline Resolution $(\AA)$ & $\begin{array}{l}60.9-2.1 \\
(2.2-2.1)^{a}\end{array}$ & $\begin{array}{l}83.7-4.3 \\
(4.4-4.3)\end{array}$ & $\begin{array}{l}117.0-4.2 \\
(4.4-4.2)\end{array}$ \\
\hline$R_{\text {p.i.m. }}(\%)$ & $3.8(67.7)$ & $5.2(51.2)$ & $5.6(66.5)$ \\
\hline $1 / \sigma$ & $23.9(1.2)$ & $18.7(1.4)$ & $19.1(1.3)$ \\
\hline $\mathrm{CC}_{1 / 2}$ & $\begin{array}{l}1(0.69) \\
99.4(98.9)\end{array}$ & $\begin{array}{l}0.94(0.52) \\
99.5(99.2)\end{array}$ & $\begin{array}{l}0.98(0.64) \\
99.9(99.9)\end{array}$ \\
\hline \multicolumn{4}{|l|}{ Completeness (\%) } \\
\hline $\begin{array}{l}\text { Redundancy } \\
\text { Refinement }\end{array}$ & $7.2(6.9)$ & $7.3(7.2)$ & $37.9(39.9)$ \\
\hline $\begin{array}{l}\text { Resolution } \\
\text { range }(\AA)\end{array}$ & $60.9-2.1$ & $83.7-4.3$ & $117.0-4.2$ \\
\hline No. reflections & 64,310 & 11,641 & 16,995 \\
\hline$R_{\text {Work }} / R_{\text {Free }}$ & $21.6 / 25.8$ & $33.6 / 35.9$ & $31.3 / 34.7$ \\
\hline \multicolumn{4}{|l|}{ No. atoms } \\
\hline Protein & 5,786 & 3818 & 6890 \\
\hline Water & 140 & 0 & 0 \\
\hline \multicolumn{4}{|l|}{$B$ factors $\left(\AA^{2}\right)$} \\
\hline Protein & 88.6 & 241.0 & 207.3 \\
\hline Water & 69.4 & - & - \\
\hline \multicolumn{4}{|l|}{ r.m.s. deviations } \\
\hline Bond length $(\AA)$ & 0.011 & 0.002 & 0.006 \\
\hline Bond angles $\left({ }^{\circ}\right)$ & 1.101 & 0.605 & 0.949 \\
\hline
\end{tabular}

Fluorescence microscopy. Strains were grown overnight in SD-Ura media (CSMUra (Sunrise Science), Yeast nitrogen base with ammonium sulfate, 2\% (v/v) glucose) at $30^{\circ} \mathrm{C}$, followed by 20 -fold dilution into fresh SC-Ura media. After growth for $4-5 \mathrm{~h}$ at $30^{\circ} \mathrm{C}$ to OD600 $\sim 0.5$, cells applied to a thin SD-Ura agar pad on a standard microscopy slide and imaged live on a DeltaVision Elite Widefield Deconvolution Microscope (GE Healthcare) using a 100x oil immersion objective with an sCMOS camera (Teledyne Photometics). Images were processed and analyzed in Image ${ }^{66}$.

Reporting summary. Further information on research design is available in the Nature Research Reporting Summary linked to this article.

\section{Data availability}

Coordinates and structure factors have been deposited in the Protein Data Bank under PDB accession codes 6X06 (Nup120 $0_{1-757}$-VHH-SAN10/11), 6 X07 (Nic96 $186-839-\mathrm{VHH}-$ SAN12), 6 X08 (Nup85 ${ }_{1-564}$-Seh1-VHH-SAN2). The cryo-ET map used for docking of the $\mathrm{Y}$ complex and nanobodies is described elsewhere ${ }^{24}$ and available from the Electron Microscopy Data Bank (EMDB) under accession number EMD-10198.

Received: 8 June 2020; Accepted: 30 October 2020; Published online: 02 December 2020

\section{References}

1. Knockenhauer, K. E. \& Schwartz, T. U. The nuclear pore complex as a flexible and dynamic gate. Cell 164, 1162-1171 (2016).

2. Schwartz, T. U. The structure inventory of the nuclear pore complex. J. Mol. Biol. 428, 1986-2000 (2016).

3. Beck, M. \& Hurt, E. The nuclear pore complex: understanding its function through structural insight. Nat. Rev. Mol. Cell Biol. 18, 73-89 (2016).

4. Hampoelz, B., Andres-Pons, A., Kastritis, P. \& Beck, M. Structure and assembly of the nuclear pore complex. Annu. Rev. Biophys. 48, 515-536 (2019) 
5. Lutzmann, M., Kunze, R., Buerer, A., Aebi, U. \& Hurt, E. Modular selfassembly of a Y-shaped multiprotein complex from seven nucleoporins. EMBO J. 21, 387-397 (2002).

6. Kabachinski, G. \& Schwartz, T. U. The nuclear pore complex - structure and function at a glance. J. Cell Sci. 128, 423-429 (2015).

7. Fernandez-Martinez, J. et al. Structure-function mapping of a heptameric module in the nuclear pore complex. J. Cell Biol. 196, 419-434 (2012).

8. Kelley, K., Knockenhauer, K. E., Kabachinski, G. \& Schwartz, T. U. Atomic structure of the Y complex of the nuclear pore. Nat. Struct. Mol. Biol. 22, 425-431 (2015).

9. Kampmann, M. \& Blobel, G. Three-dimensional structure and flexibility of a membrane-coating module of the nuclear pore complex. Nat. Struct. Mol. Biol. 16, 782-788 (2009).

10. Stuwe, T. et al. Architecture of the nuclear pore complex coat. Science 347, 1148-1152 (2015).

11. Vollmer, B. \& Antonin, W. The diverse roles of the Nup93/Nic96 complex proteins - structural scaffolds of the nuclear pore complex with additional cellular functions. Biol. Chem. 395, 515-528 (2014).

12. Grandi, P., Schlaich, N., Tekotte, H. \& Hurt, E. C. Functional interaction of Nic96p with a core nucleoporin complex consisting of Nsplp, Nup49p and a novel protein Nup57p. EMBO J. 14, 76-87 (1995).

13. Alber, F. et al. The molecular architecture of the nuclear pore complex. Nature 450, 695-701 (2007).

14. Chug, H., Trakhanov, S., Hülsmann, B. B., Pleiner, T. \& Görlich, D. Crystal structure of the metazoan Nup62 Nup58・Nup54 nucleoporin complex. Science 350, 106-110 (2015)

15. Stuwe, T. et al. Architecture of the fungal nuclear pore inner ring complex. Science 350, 56-64 (2015).

16. Fischer, J., Teimer, R., Amlacher, S., Kunze, R. \& Hurt, E. Linker Nups connect the nuclear pore complex inner ring with the outer ring and transport channel. Nat. Struct. Mol. Biol. 22, 774-781 (2015).

17. Brohawn, S. G., Partridge, J. R., Whittle, J. R. R. \& Schwartz, T. U. The nuclear pore complex has entered the atomic age. Structure 17, 1156-1168 (2009).

18. Brohawn, S. G., Leksa, N. C., Spear, E. D., Rajashankar, K. R. \& Schwartz, T. $\mathrm{U}$. Structural evidence for common ancestry of the nuclear pore complex and vesicle coats. Sci. N. Y. N. Y. 322, 1369-1373 (2008).

19. Lin, D. H. \& Hoelz, A. The structure of the nuclear pore complex (an update). Annu. Rev. Biochem 88, 725-783 (2019).

20. Nordeen, S. A., Turman, D. L. \& Schwartz, T. U. Structure of the yeast Nup84Nup133 complex details flexibility and reveals universal conservation of the membrane anchoring ALPS motif. Preprint at https://doi.org/10.1101/ 2020.06.19.161133 (2020)

21. Eibauer, M. et al. Structure and gating of the nuclear pore complex. Nat. Commun. 6, 7532 (2015)

22. Appen, Avon et al. In situ structural analysis of the human nuclear pore complex. Nature 526, 140-143 (2015).

23. Kim, S. J. et al. Integrative structure and functional anatomy of a nuclear pore complex. Nature 555, 475-482 (2018).

24. Allegretti, M. et al. In-cell architecture of the nuclear pore and snapshots of its turnover. Nature 586, 796-800 (2020).

25. Bui, K. H. et al. Integrated structural analysis of the human nuclear pore complex scaffold. Cell 155, 1233-1243 (2013).

26. Kosinski, J. et al. Molecular architecture of the inner ring scaffold of the human nuclear pore complex. Science 352, 363-365 (2016).

27. Lin, D. H. et al. Architecture of the symmetric core of the nuclear pore. Science 352, aaf1015 (2016).

28. Rajoo, S., Vallotton, P., Onischenko, E. \& Weis, K. Stoichiometry and compositional plasticity of the yeast nuclear pore complex revealed by quantitative fluorescence microscopy. Proc. Natl Acad. Sci. USA 115, E3969-E3977 (2018).

29. Seo, H. S. et al. Structural and functional analysis of Nup120 suggests ring formation of the Nup84 complex. Proc. Natl Acad. Sci. USA 106, 14281-14286 (2009).

30. Fernandez-Martinez, J. et al. Structure and function of the nuclear pore complex cytoplasmic mRNA export platform. Cell 167, 1215-1228 (2016).

31. Ingram, J. R., Schmidt, F. I. \& Ploegh, H. L. Exploiting nanobodies' singular traits. Annu. Rev. Immunol. 36, 695-715 (2018).

32. Helma, J., Cardoso, M. C., Muyldermans, S. \& Leonhardt, H. Nanobodies and recombinant binders in cell biology. J. Cell Biol. 209, 633-644 (2015).

33. McMahon, C. et al. Yeast surface display platform for rapid discovery of conformationally selective nanobodies. Nat. Struct. Mol. Biol. 25, 289-296 (2018).

34. Zimmermann, I. et al. Synthetic single domain antibodies for the conformational trapping of membrane proteins. eLife 7, e34317 (2018).

35. Brohawn, S. G. \& Schwartz, T. U. Molecular architecture of the Nup84 Nup145C-Sec13 edge element in the nuclear pore complex lattice. Nat. Struct. Mol. Biol. 16, 1173-1177 (2009).
36. Beckett, D., Kovaleva, E. \& Schatz, P. J. A minimal peptide substrate in biotin holoenzyme synthetase-catalyzed biotinylation. Protein Sci. 8, 921-929 (2008).

37. Mitchell, L. S. \& Colwell, L. J. Comparative analysis of nanobody sequence and structure data. Proteins Struct. Funct. Bioinform 86, 697-706 (2018).

38. Desmyter, A., Spinelli, S., Roussel, A. \& Cambillau, C. Camelid nanobodies: killing two birds with one stone. Curr. Opin. Struct. Biol. 32, 1-8 (2015).

39. Waterhouse, A. et al. SWISS-MODEL: homology modelling of protein structures and complexes. Nucleic Acids Res. 46, W296-W303 (2018).

40. Leksa, N. C., Brohawn, S. G. \& Schwartz, T. U. The structure of the scaffold nucleoporin Nup120 reveals a new and unexpected domain architecture. Structure 17, 1082-1091 (2009).

41. Braun, M. B. et al. Peptides in headlock-a novel high-affinity and versatile peptide-binding nanobody for proteomics and microscopy. Sci. Rep.-uk 6, 19211 (2016).

42. Götzke, H. et al. The ALFA-tag is a highly versatile tool for nanobody-based bioscience applications. Nat. Commun. 10, 4403 (2019).

43. Ling, J. et al. A nanobody that recognizes a 14-residue peptide epitope in the E2 ubiquitin-conjugating enzyme UBC6e modulates its activity. Mol. Immunol. 114, 513-523 (2019).

44. Cheloha, R. W., Harmand, T. J., Wijne, C., Schwartz, T. U. \& Ploegh, H. L. Exploring cellular biochemistry with nanobodies. J. Biol. Chem. https://doi. org/10.1074/jbc.rev120.012960 (2020).

45. Jeudy, S. \& Schwartz, T. U. Crystal structure of nucleoporin Nic96 reveals a novel, intricate helical domain architecture. J. Biol. Chem. 282, 34904-34912 (2007).

46. Schrader, N. et al. Structural basis of the Nic96 subcomplex organization in the nuclear pore channel. Mol. Cell 29, 46-55 (2008).

47. Dokudovskaya, S. et al. A conserved coatomer-related complex containing Sec13 and Seh1 dynamically associates with the vacuole in Saccharomyces cerevisiae. Mol. Cell Proteom. 10, M110.006478 (2011).

48. Whittle, J. R. R. \& Schwartz, T. U. Structure of the Sec13-Sec16 edge element a template for assembly of the COPII vesicle coat. J. Cell Biol. 190, 347-361 (2010).

49. Miller, E. A. \& Schekman, R. COPII - a flexible vesicle formation system. Curr Opin. Cell Biol. 25, 420-427 (2013).

50. Antonny, B. Mechanisms of membrane curvature sensing. Annu. Rev. Biochem. 80, 101-123 (2011).

51. Drin, G. et al. A general amphipathic a-helical motif for sensing membrane curvature. Nat. Struct. Mol. Biol. 14, 138-146 (2007).

52. Kim, S. J. et al. Integrative structure-function mapping of the nucleoporin Nup133 suggests a conserved mechanism for membrane anchoring of the nuclear pore complex. Mol. Cell Proteom. 13, 2911-2926 (2014).

53. Ori, A. et al. Cell type-specific nuclear pores: a case in point for contextdependent stoichiometry of molecular machines. Mol. Syst. Biol. 9, 648 (2013).

54. Stanley, G. J., Fassati, A. \& Hoogenboom, B. W. Atomic force microscopy reveals structural variability amongst nuclear pore complexes. Life Sci. Alliance 1, e201800142 (2018).

55. Lupu, F., Alves, A., Anderson, K., Doye, V. \& Lacy, E. Nuclear pore composition regulates neural stem/progenitor cell differentiation in the mouse embryo. Dev. Cell 14, 831-842 (2008).

56. Onischenko, E. et al. Natively unfolded FG repeats stabilize the structure of the nuclear pore complex. Cell 171, 904-917 (2017).

57. Frey, S. \& Görlich, D. A new set of highly efficient, tag-cleaving proteases for purifying recombinant proteins. J. Chromatogr. A 1337, 95-105 (2014).

58. Andersen, K. R., Leksa, N. C. \& Schwartz, T. U. Optimized E. coli expression strain LOBSTR eliminates common contaminants from His-tag purification. Proteins 81, 1857-1861 (2013).

59. Ingram, J. R. et al. Allosteric activation of apicomplexan calcium-dependent protein kinases. Proc. Natl Acad. Sci. USA 112, E4975-E4984 (2015).

60. Morin, A. et al. Collaboration gets the most out of software. eLife 2, e01456 (2013).

61. Otwinowski, Z. \& Minor, W. Processing of X-ray diffraction data collected in oscillation mode. Methods Enzymol. 276, 307-326 (1997).

62. Emsley, P., Lohkamp, B., Scott, W. G. \& Cowtan, K. Features and development of Coot. Acta Crystallogr. Sect. D. Biol. Crystallogr. 66, 486-501 (2010).

63. Adams, P. D. et al. PHENIX: a comprehensive Python-based system for macromolecular structure solution. Acta Crystallogr. Sect. D. Biol. Crystallogr. 66, 213-221 (2010)

64. Decanniere, K. et al. A single-domain antibody fragment in complex with RNase A: non-canonical loop structures and nanomolar affinity using two CDR loops. Structure 7, 361-370 (1999).

65. Longtine, M. S. et al. Additional modules for versatile and economical PCR based gene deletion and modification in Saccharomyces cerevisiae. Yeast 14, 953-961 (1998).

66. Schindelin, J. et al. Fiji: an open-source platform for biological-image analysis. Nat. Methods 9, 676-682 (2012). 


\section{Acknowledgements}

We thank Nina C. Leksa and Xuanzong Guo for their initial work on the Nup120 structure and members of the Schwartz lab for discussions on the experiments and manuscript. We thank Karsten Weis for advice on fluorescence microscopy and experiments in yeast. The research was supported by the US NIH under grant number R01GM77537 (K.E.K., K.A., and T.U.S.) and T32GM007287 (S.A.N.). J.R.I. and H.P. were supported by an NIH Pioneer award. The X-ray crystallography work was conducted at the APS NE-CAT beamlines, which are supported by NIH award GM103403. Use of the APS is supported by the US Department of Energy, Office of Basic Energy Sciences, under contract no. DE-AC02-06CH11357.

\section{Author contributions}

S.A.N. and T.U.S. designed the study. S.A.N. performed the experiments. K.A. solved the structure of Nic96 $186-839^{-V H H}-\mathrm{SAN} 12$ and K.E.K. solved the structure of Nup120 $1-757^{-}$ VHH-SAN10/11. J.R.I. and H.P. conducted the alpaca immunization experiments and generation of the phagemid library. S.A.N. and T.U.S. interpreted the results and wrote the manuscript with input from K.A., K.E.K., and H.P.

\section{Competing interests}

The authors declare no competing interests.

\section{Additional information}

Supplementary information is available for this paper at https://doi.org/10.1038/s41467020-19884-6.
Correspondence and requests for materials should be addressed to T.U.S.

Peer review information Nature Communications thanks André Hoelz and Jan Steyaert for their contribution to the peer review of this work. Peer reviewer reports are available

Reprints and permission information is available at http://www.nature.com/reprints

Publisher's note Springer Nature remains neutral with regard to jurisdictional claims in published maps and institutional affiliations.

(c) (i) Open Access This article is licensed under a Creative Commons Attribution 4.0 International License, which permits use, sharing, adaptation, distribution and reproduction in any medium or format, as long as you give appropriate credit to the original author(s) and the source, provide a link to the Creative Commons license, and indicate if changes were made. The images or other third party material in this article are included in the article's Creative Commons license, unless indicated otherwise in a credit line to the material. If material is not included in the article's Creative Commons license and your intended use is not permitted by statutory regulation or exceeds the permitted use, you will need to obtain permission directly from the copyright holder. To view a copy of this license, visit http://creativecommons.org/ licenses/by/4.0/.

(C) The Author(s) 2020 